\title{
A transcriptome analysis reveals a role for the indole GLS-linked auxin biosynthesis in secondary dormancy in rapeseed (Brassica napus L.)
}

Lei Liu ${ }^{1,2+}$, Fuxia Liu ${ }^{1,2+}$, Jinfang Chu ${ }^{4 \dagger}$, Xin Yi ${ }^{1,2}$, Wenqi Fan ${ }^{1,2,3}$, Tang Tang ${ }^{1,2}$, Guimin Chen ${ }^{1,2}$, Qiuhuan Guo ${ }^{4}$ and Xiangxiang Zhao ${ }^{1,2^{*}}$

\begin{abstract}
Background: Brassica napus L. has little or no primary dormancy, but exhibits great variation in secondary dormancy. Secondary dormancy potential in oilseed rape can lead to the emergence of volunteer plants that cause genetic contamination, reduced quality and biosafety issues. However, the mechanisms underlying secondary dormancy are poorly understood. In this study, cultivars Huaiyou-WSD-H2 (H) and Huaiyou-SSD-V1 (V), which exhibit low (approximately 5\%) and high (approximately 95\%) secondary dormancy rate, respectively, were identified. Four samples, before ( $\mathrm{Hb}$ and $\mathrm{Vb}$ ) and after ( $\mathrm{Ha}$ and $\mathrm{Va}$ ) secondary dormancy induction by polyethylene glycol (PEG), were collected to identify the candidate genes involved in secondary dormancy via comparative transcriptome profile analysis.

Results: A total of 998 differentially expressed genes (DEGs), which are mainly involved in secondary metabolism, transcriptional regulation, protein modification and signaling pathways, were then detected. Among these DEGs, the expression levels of those involved in the sulfur-rich indole glucosinolate (GLS)-linked auxin biosynthesis pathway were markedly upregulated in the dormant seeds (Va), which were validated by qRT-PCR and subsequently confirmed via detection of altered concentrations of indole-3-acetic acid (IAA), IAA conjugates and precursors. Furthermore, exogenous IAA applications to cultivar H enhanced secondary dormancy.

Conclusion: This study first (to our knowledge) elucidated that indole GLS-linked auxin biosynthesis is enhanced during secondary dormancy induced by PEG, which provides valuable information concerning secondary dormancy and expands the current understanding of the role of auxin in rapeseed.
\end{abstract}

Keywords: Brassica napus, Secondary dormancy, Germination, Volunteer plant, Phytohormone, RNA-seq analysis

\section{Background}

Seed dormancy is an important adaptive trait of seed plants and is influenced by both genetic factors and environmental cues to ensure timely germination for propagation $[1,2]$. The classification of primary and secondary dormancy is widely accepted based on the occurrence time. Primary

\footnotetext{
*Correspondence: xxzhao2013@163.com

${ }^{+}$Lei Liu, Fuxia Liu and Jinfang Chu contributed equally to this work.

'Jiangsu Key Laboratory for Eco-agriculture Biotechnology around Hongze Lake, Huaiyin Normal University, Huai'an 223300, China

${ }^{2}$ Jiangsu Collaborative Innovation Center of Regional Modern Agriculture and Environment Protection, Huaiyin Normal University, Huai'an 223300, China Full list of author information is available at the end of the article
}

dormancy, which has been thoroughly studied in model plants, is induced and maintained during seed development on the mother plant, and peaks when the seeds mature and are released by after-ripening [3]. By contrast, secondary dormancy, which is mainly responsible for dormancy cycling, is defined as the inability of mature and non-dormant seeds to germinate under adverse conditions (anoxia, high temperature and/or drought) $[4,5]$. Once primary dormancy is released and adverse conditions start to take effect, secondary dormancy of dispersed seeds that originate from seed shedding in the field may be induced. Then the secondary dormant seeds involve repeated induction and

(c) The Author(s). 2019 Open Access This article is distributed under the terms of the Creative Commons Attribution 4.0 International License (http://creativecommons.org/licenses/by/4.0/), which permits unrestricted use, distribution, and 
termination in response to environmental conditions until the proper conditions for germination are met [6].

Brassica napus L. is one of the most important cultivated oilseed crops worldwide. Oilseed rape seeds undergo little or no primary dormancy $[7,8]$, but exhibit different secondary dormancy potential $[5,9]$. Before and during the harvest of the seeds, seed losses can reach up to 10 , 000 seeds per $\mathrm{m}^{2}$ because of silique shattering [10]. Under adverse conditions, the shed seeds can be induced into secondary dormancy and remain alive for 10 years or even longer, thereby increasing the size of the seed bank in the soil [11-13]. Once encountering favorable conditions, the dormant seeds will germinate to emerge as a weed (if among other crop species) or as volunteer plant (among oilseed rape crops) during subsequent crop cultivation. The volunteer plants might cross-pollinate with the subsequent crop plants and reduce seed quality [7, 14]. Moreover, the volunteers could lead to biosafety issues by cross pollination given the persistence of genetically modified oilseed rape in the seed bank $[15,16]$. In this situation, genetic improvement resulting in plants with low or no secondary dormancy will help efficiently reduce the soil seed bank to avoid biosafety risks. Therefore, research on the genetic basis and mechanism underlying secondary dormancy is of great theoretical and practical significance.

Oilseed rape shows large and significant variation in its capacity for secondary dormancy $(<60 \%$ for winter varieties, $<80 \%$ for spring varieties), and the trait is highly heritable $[8,10,16,17]$. The contributions of genotype, seed size and harvest time to secondary dormancy have been quantified and the genotype contribution ranged from 44 to $82 \%$ of the total variation [7], suggesting that genotype was the decisive factor in secondary dormancy. With PEG6000 treatment for 2 weeks in the dark $[5,10,18]$, four quantitative trait loci (QTL) for secondary dormancy located on chromosomes A5, C3, C5 and C8 were detected via a doubled haploid (DH) population derived from a cross between the cultivar Express 617 (30.4\% secondary dormancy) and the accession R53 (61.7\% secondary dormancy), which explained $35 \%$ of the phenotypic variation [16].

Previous studies have indicated that the molecular mechanism underlying primary dormancy relies on the signal integration of both hormone (abscisic acid (ABA) / gibberellic acid (GA) balance) and gene expression (DOG1) thresholds [19-21]. ABA and GA have been recognized as the predominant hormones that antagonistically regulate seed dormancy [22]. Recently, increasing evidence have shown that another phytohormone, auxin, is also crucial for primary dormancy in Arabidopsis, although its effects are somewhat debatable [23-26]. ABA plays crucial roles in rapeseed secondary dormancy [7]; however, whether auxin plays pivotal roles remains unknown. Furthermore, the mechanism by which secondary dormancy is induced and maintained is poorly understood.

RNA sequencing (RNA-seq) has become a very useful tool for understanding the relationships between the underlying mechanism of developmental processes and gene expression profiles at the whole-genome level in Brassica napus, including the genetic basis of salt tolerance [27], that of root length [28], and the effects of night temperature on storage lipids [29]. In the present study, we performed RNA-seq using two representative cultivars (approximately 95 and 5\% secondary dormancy, respectively) to detect the differentially expressed genes (DEGs) responsible for secondary dormancy. This study aimed to determine the significant transcriptome changes in secondary dormant and non-dormant seeds and revealed a role of indole glucosinolate (GLS)-linked auxin biosynthesis in secondary dormancy.

\section{Methods \\ Plant materials}

Two cultivars of winter type rapeseed (Brassica napus L.) were used in this study, showing significant difference in secondary dormancy potential. The low secondary dormancy cultivar namely Huaiyou-WSD-H2 (abbreviated as $\mathrm{H})$ showed secondary dormancy rate lower than $5 \%$ induced by PEG and the high secondary dormancy cultivar namely Huaiyou-SSD-V1 (abbreviated as V) showed secondary dormancy higher than $90 \%$ (Table 1) induced by PEG. Besides, the two selected cultivars are also different in many seed quality traits (Additional file 2: Table S12). Both cultivars were screened from those of advanced generations bred by Dr. Zhao's research group in Huaiyin Normal University.

\section{Induction of secondary dormancy}

The rapeseed cultivars $\mathrm{H}$ and $\mathrm{V}$ were planted in the experimental field in Biotechnology Park of Huaiyin Normal University, with each cultivar forming a plot of $30 \mathrm{~m}^{2}$. Individual plants of each cultivar were bagged separately for self-pollination at flowering stage. After maturation the selfed seeds of each cultivar were collected, dried and stored under constant temperatures at $25^{\circ} \mathrm{C}$ for secondary dormancy testing.

Secondary seed dormancy was induced in accordance with the protocol described by Gruber et al. [17] with slight modifications. Dry seeds of $\mathrm{H}(4 \times 100)$ and V $(4 \times$ 100) were placed in four Petri dishes (100 seeds per Petri dish) layered with two pieces of filter paper for one biological replicate. Then $8 \mathrm{ml}$ of PEG6000 solution were added to the dishes with an initial osmotic potential of 1.5 $\mathrm{MPa}$ and put into a cardboard box. The cardboard boxes were then wrapped with black cloth and placed in a growth chamber (MLR-351H, SANYO) in the dark at $20^{\circ} \mathrm{C}$ for two weeks. Afterward, the seeds were washed 
Table 1 Statistical analyses of secondary dormancy ratio of cultivars $\mathrm{H}$ and $\mathrm{V}$ from three independent replicates

\begin{tabular}{|c|c|c|c|c|c|c|c|c|c|c|c|c|c|c|c|c|c|c|c|}
\hline \multirow[t]{3}{*}{ Year } & \multirow[t]{3}{*}{ CV. } & \multicolumn{5}{|c|}{ Germination in water (Control) } & \multicolumn{13}{|c|}{ Secondary dormancy after PEG induction } \\
\hline & & \multicolumn{4}{|c|}{ No. of germinated seeds } & \multirow{2}{*}{$\begin{array}{l}\text { Rate (\%) } \\
\text { Average }\end{array}$} & \multicolumn{4}{|c|}{ No. of non-germinated seeds } & \multicolumn{4}{|c|}{$\begin{array}{l}\text { No. of germinated seeds after } \\
\text { dormancy release }\end{array}$} & \multicolumn{5}{|c|}{ Secondary dormancy rate (\%) } \\
\hline & & I & $\|$ & III & IV & & I & $\|$ & III & IV & I & $\|$ & III & IV & I & $\|$ & III & IV & Average $^{a}$ \\
\hline \multirow[t]{2}{*}{2015} & $\mathrm{H}$ & 100 & 100 & 100 & 100 & 100 & 4 & 5 & 2 & 3 & 4 & 5 & 2 & 3 & 4 & 5 & 2 & 3 & $3.5 \pm 1.3$ \\
\hline & V & 100 & 100 & 100 & 100 & 100 & 92 & 91 & 96 & 95 & 92 & 91 & 96 & 95 & 92 & 91 & 96 & 95 & $93.5 \pm 2.4$ \\
\hline \multirow[t]{2}{*}{2016} & $\mathrm{H}$ & 100 & 100 & 100 & 100 & 100 & 0 & 2 & 4 & 1 & 0 & 2 & 4 & 1 & 0 & 2 & 4 & 1 & $1.8 \pm 1.7$ \\
\hline & V & 100 & 100 & 100 & 100 & 100 & 100 & 99 & 97 & 95 & 100 & 99 & 97 & 95 & 100 & 99 & 97 & 95 & $97.8 \pm 2.2$ \\
\hline \multirow[t]{2}{*}{2017} & $\mathrm{H}$ & 100 & 100 & 100 & 100 & 100 & 4 & 3 & 3 & 3 & 4 & 3 & 3 & 3 & 4 & 3 & 3 & 3 & $3.3 \pm 0.5$ \\
\hline & V & 100 & 100 & 100 & 100 & 100 & 94 & 95 & 95 & 98 & 94 & 95 & 95 & 98 & 94 & 95 & 95 & 98 & $95.5 \pm 1.7$ \\
\hline
\end{tabular}

$\mathrm{H}$ represents the cultivar Huaiyou-WSD-H2, and $\mathrm{V}$ denotes the cultivar Huaiyou-SSD-V1

Cv. represents cultivar

I, II, III and IV denote the number of four dishes in one biological replicate, respectively

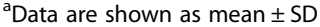

with water under green light $(500-600 \mathrm{~nm})$ to remove any PEG and immediately transferred into new Petri dishes supplemented with $6 \mathrm{ml}$ of pure water. Then the Petri dishes were put back to the card boxes with black cloth and transferred to the chamber for germination. Eight days later, the germinated seeds were removed and the remaining seeds were placed in chamber under alternating light and temperature conditions $\left(12 \mathrm{~h}\right.$ dark at $3{ }^{\circ} \mathrm{C}$ and $12 \mathrm{~h}$ light at $30^{\circ} \mathrm{C}$ ) to release dormancy to test the seed viability. The germinated seeds under the alternating conditions were considered to be secondary dormant seeds and the rest were unviable. Secondary dormancy rate was calculated as: Secondary dormancy $(\%)=$ secondary dormant seeds "100 / (100-unviable seeds). Simultaneously, both stains $\mathrm{H}$ and $\mathrm{V}$ imbibed in water instead of PEG were used as a control.

Secondary dormancy rates of both cultivars $\mathrm{H}$ and $\mathrm{V}$ were calculated in the consecutive three years from year 2015 to 2017.

\section{Sample harvest and RNA extraction}

For one biological replicate, four samples, including mature seeds of cultivar $\mathrm{H}$ and $\mathrm{V}(\mathrm{Hb}$ and $\mathrm{Vb})$ and PEG6000induced seeds ( $\mathrm{Ha}$ and $\mathrm{Va}$ ), were collected. The details per replicate are as follows: During secondary dormancy induction, seeds of $\mathrm{H}$ and $\mathrm{V}$ were imbibed in PEG6000 with an initial osmotic potential of $-1.5 \mathrm{MPa}$ for two weeks. At that time, seeds of $\mathrm{H}$, which were not induced into secondary dormancy, were sampled and referred to Ha. Seeds of $\mathrm{V}$ were washed with water under green light to remove any PEG and immediately transferred into new Petri dishes supplemented with $6 \mathrm{ml}$ of pure water to germinate in the dark for another 8 days. Afterwards, the germinated seeds were removed and the remaining seeds induced into secondary dormancy were sampled and referred to Va. Meantime, mature seeds of $\mathrm{H}$ and $\mathrm{V}$, which were not subjected to PEG treatment, were sampled and referred to $\mathrm{Hb}$ and $\mathrm{Vb}$ respectively. Three independent replicates were conducted and 12 samples in total were harvested for RNA extraction.

The total RNA was extracted from $0.1 \mathrm{~g}$ of mature seeds per sample using an Aidlab RN38 RNA extraction kit (Aidlab, China) according to the manufacturer's instructions with slight modifications by adding $300 \mu \mathrm{l}$ chloroform to centrifuge before the second step.

\section{RNA-seq experiments}

The quality of the total RNA was analysed using an Agilent 2100 Bioanalyzer with threshold value of RNA integrity number $(\mathrm{RIN}) \geq 7.5$. The RNA $(0.1-0.4 \mu \mathrm{g})$ was then precisely quantified using a QUBIT RNA Assay Kit (Invitrogen, America). mRNA was purified from the total RNA using magnetic beads with oligo $(\mathrm{dT})$ primers and then fragmented into $120-210 \mathrm{bp}$. First-strand cDNA was synthesized via a SuperScript II Kit (Invitrogen, America) and the second strand was synthesized by DNA polymerase I. After end repair and $3^{\prime}$ end adenylation, the indexed adapter was ligated to both ends using T4 DNA ligase. The cDNA was then purified twice by AMPure XP Beads (Beckman Coulter, America) to eliminate redundant adapters and amplified via PCR. Finally, a gel purification procedure was performed to select the fragments ranging from 300 to $350 \mathrm{bp}$ in size to produce a paired-end library. The fragment size was controlled via an Agilent 2100 Bioanalyzer, and the yield was assessed via a KAPA qPCR Kit (Kapa Biosystems, America). A $10 \mu$ library ( $2 \mathrm{nM})$ was fixed onto cBot (Illumina, America) and sequenced on an Illumina X10 platform with 150 bp paired-end reads by CapitalBio Corporation, Beijing, China.

RNA-Seq data processing and gene expression annotation RNA-seq raw reads were processed using an NGS-QC toolkit [30], after which low-quality and adaptercontaminated sequences were discarded. The paired high-quality reads were then aligned with the reference genome of Brassica napus (http://www.genoscope.cns. 
fr/brassicanapus/data/) via HISAT [31]. To obtain more functional annotations, all the detected genes were blasted against the Arabidopsis genome (The Arabidopsis Information Resource (TAIR), http://www.arabidopsis.org/Blast/index.jsp) using BLASTN. The functions of the unigenes were annotated via BLASTX searches against the non-redundant (NR), SwissProt, and Uniprot databases $(\mathrm{E}<1 \mathrm{e}-5)$. The Gene Ontology $(\mathrm{GO})$ annotations were determined using Blast2GO with the UniProtKB/SwissProt database. Pathway annotations were performed to understand gene functions via BLASTX of the Kyoto Encyclopedia of Genes and Genomes (KEGG) database. For GO and KEGG enrichment, a corrected $p \leq 0.05$ value was set as the threshold. The MapMan software (http://mapman.gabipd. org/home, 19.11.2010) was used to provide a graphical overview of the metabolic and regulatory pathways.

\section{Quantification of gene expression levels and analysis of DEGs}

Gene expression levels were calculated using the fragments per kilobase per million fragments (FPKM) method [32] via Cuffquant [33] and Cuffnorm softwares [33]. Differential gene expression analysis was carried out by Cuffdiff [34] software based on two criteria: (a) an absolute of $\log _{2}$ fold-change (FC) $\geq 1$ and (b) a q value (false discovery rate $(\mathrm{FDR})) \leq 0.001$.

\section{Real-time qRT-PCR analysis of DEGs}

To validate the DEGs identified from RNA-seq analysis, real-time qRT-PCR was conducted. The total RNA was isolated from newly treated samples (not those used for RNA-seq). $1 \mu \mathrm{g}$ of total RNA free of DNA contamination was used to synthesize first-strand cDNA, which was synthesized with oligo (dT) primers via a the Revert Aid First Strand cDNA Synthesis Kit (K1622, Thermo). Considering the allopolyploid oilseed rape genome, we download all loci sequences of the same gene which is to be detected according to their accession numbers (http://brassicadb.org/brad/datasets/pub/Genomes/Brassica_napus/). Gene-specific primers were designed according to 1) the same sequences from all loci of the same gene and 2) unique sequences to the gene (http:// bioinfo.ut.ee/primer3/; Table 2). The expression was measured from all loci of the same gene. PCR products were confirmed to be specific by melt curve analysis and be a single band by gel electrophoresis. The reaction mixtures were prepared with a SYBR PrimeScript ${ }^{\text {tox }}$ RTPCR Kit (RR820A, TaKaRa) and then loaded into a BioRad CFX Manager instrument. The PCR procedure was as follows: $95^{\circ} \mathrm{C}$ for $5 \mathrm{~s}$ followed by 40 cycles of $95^{\circ} \mathrm{C}$ for $3 \mathrm{~s}$ and $60^{\circ} \mathrm{C}$ for $30 \mathrm{~s}$. Three biological replicates per sample and three technical replicates were conducted, and the relative expression levels were calculated using the $2^{-\triangle \Delta C T}$ method [35]. As an internal control, BnaCAT1 (BanC07g15270D) was used to normalize the gene expression (Additional file 2: Table S3).

\section{Analysis of the content of auxin intermediates}

Analysis of IAA, tryptophan, IAA-Asp, and IAA-Glu:

Samples for IAA, tryptophan, IAA-Asp, and IAA-Glu analysis were extracted and purified as described in the method reported by $\mathrm{Fu}$ et al. [36] with some modifications: Dry seeds or seeds subjected to PEG6000 treatment (200 $\mathrm{mg}, \mathrm{DW}$ ) were ground to fine powder in liquid nitrogen, then extracted with $80 \% \mathrm{MeOH}$ containing internal standards $\left(10 \mathrm{ng}{ }^{13} \mathrm{C}_{11}, 10 \mathrm{ng}{ }^{15} \mathrm{~N}_{2}\right.$-trp and $2.5 \mathrm{ng}{ }^{2} \mathrm{H}_{2}$-IAA) and at $-20^{\circ} \mathrm{C}$ for $16 \mathrm{~h}$. After centrifugation, the supernatant was collected and evaporated to dryness. The dried residue was reconstituted in $5 \% \mathrm{NH}_{4} \mathrm{OH}$, and loaded onto the Oasis MAX (Waters) cartridge. The cartridge was sequentially washed with $5 \% \mathrm{NH}_{4} \mathrm{OH}$, water and $\mathrm{MeOH}$, and then was eluted with $5 \%$ formic acid (FA) in $\mathrm{MeOH}$. The eluate was evaporated and re-dissolved in $80 \% \mathrm{MeOH}$ for LCMS/MS analysis. LC-MS/MS analysis was performed on a UPLC system (Waters) coupled to the 6500 Qtrap system (AB SCIEX). LC separation performed a BEH C18 column $(1.7 \mathrm{~mm}, 2.1 \times 100 \mathrm{~mm}$; Waters) with mobile phase $0.1 \%$ FA (A) and acetonitrile (ACN) (B). The gradient was set with initial $2 \% \mathrm{~B}$ and increased to $50 \% \mathrm{~B}$ within $6 \mathrm{~min}$ at a flow rate of $0.3 \mathrm{~mL} / \mathrm{min}$. Tryptophan, IAA, IAA-Asp, and IAA-Glu were detected in positive ionization and MRM mode with transitions 205/188, 176/130, 291/130, and 305/ 130 , respectively. ${ }^{2} \mathrm{H}_{2}$-IAA was used as an internal standard to quantify IAA, IAA-Asp, and IAA-Glu, and ${ }^{13} \mathrm{C}_{11},{ }^{15} \mathrm{~N}_{2}$ trp was used to quantify tryptophan level.

Analysis of indole-3-pyruvic acid (IPyA):

IPyA analysis was performed as described by Mashiguchi et al. [37] with slight modifications: About $100 \mathrm{mg}$ of seeds was ground to fine powder in liquid nitrogen and was homogenized in methanol. The extracts were centrifuged and the supernatant was transferred to a glass tube. Three extractions were done quickly and the supernatants were combined. 2,4-dinitropjenylhydrazine $(\mathrm{DNPH})$ and $1 \mathrm{~N} \mathrm{HCl}$ were added to the extract and reacted overnight at $37^{\circ} \mathrm{C}$. The amount of DNPH added to the extract was $1 \mu \mathrm{g}$ DNPH per $1 \mathrm{mg}$ fresh weight sample. After precolumn derivatization, distilled water was added to the same volume of extract and then loaded onto an Oasis MCX cartridge (Waters). The cartridge was washed with $50 \%$ methanol and eluted with the $80 \% \mathrm{MeOH}$, and the eluate was evaporated to dryness. Finally, the dried DNPH-IPA was methylated with diethyl ether and diazomethane at room temperature to get DM-IPA. DM-IPA was dissolved into 50\% acetonitrile for UPLC-MS/MS analysis.LC-MS/MS analysis was performed on a UPLC system (Waters) coupled to 
the 6500 Qtrap system (AB SCIEX). LC separation used a BEH C18 column $(1.7 \mathrm{~mm}, 2.1 \times 100 \mathrm{~mm}$; Waters $)$ with mobile phase $0.05 \%$ acetic acid (A) and $0.05 \%$ acetic acid/ACN (B). The gradient was set with initial 5\% B and increased to $60 \% \mathrm{~B}$ within $8 \mathrm{~min}$ at a flow rate of $0.3 \mathrm{~mL} / \mathrm{min}$, then increased to $95 \% \mathrm{~B}$ for $13 \mathrm{~min}$. DMIPA was detected in negative ionization and MRM mode with transition 396/152. The level of IPyA was quantitatively analyzed by an external standard curve.

\section{Germination assays of exogenous auxin treatment}

Germination assays were performed by placing rape seeds in Petri dishes lined with two layers of filter paper soaked in water or IAA solution $(10 \mathrm{mg} / \mathrm{L}, 100 \mathrm{mg} / \mathrm{L}$, $500 \mathrm{mg} / \mathrm{L}$ ) for the indicated time at $20^{\circ} \mathrm{C}$ in the dark, respectively. The IAA (I2886, Sigma) solution was first dissolved in ethanol and then in water to make the final concentration $(10 \mathrm{mg} / \mathrm{L}, 100 \mathrm{mg} / \mathrm{L}, 500 \mathrm{mg} / \mathrm{L})$. A seed was considered as germinated when its radical protruded through the seed coat. The non-germinated seeds after 8 days of imbibition were recorded to calculate the secondary dormancy ratio. Results from three independent replicates were obtained.

\section{Results}

Characterization of secondary dormancy potential in cultivars $\mathrm{H}$ and $\mathrm{V}$

To isolate the candidate genes underlying secondary dormancy in rapeseed, two advanced generation cultivars, namely Huaiyou-WSD-H2 (abbreviated as $\mathrm{H}$ hereinafter) and Huaiyou-SSD-V1 (abbreviated as V hereinafter), were identified as the low and high representative genotypes, respectively. To test the seed viability, seeds of both $\mathrm{H}$ (Fig. 1a) and V (Fig. 1b) totally

Table 2 Gene ID and gene-specific primers for qRT-PCR validation

\begin{tabular}{|c|c|c|c|}
\hline Gene ID & Forward primer & Reverse primer & Arabidopsis homolog \\
\hline BnaA03g01690D & GCAGGAGACATATTCCAGATTGTG & ATCGACCAACATGATGTGCTCAG & ASA1 \\
\hline BnaC08g25400D & CCAACAACACCAACAGAGAGAATG & GACTTTGTGAGACTCTCAAGCTCC & TSA1 \\
\hline BnaA10g09090D & CTCACTCCATCAGTGCAGGATTG & GTTCAACACGACTCTGCTTCCG & TSB1 \\
\hline BnaA01g34610D & CTCAACGAGTGCTCCGAAGTTAC & GACCATAACCAACGGTTTACACAG & CYP79B2 \\
\hline BnaA08g04520D & GAGAAACCATAGCAGACGCGAAG & СTCTACCATTGCGACTCCAAGAT & CYP83B1 \\
\hline BnaA07g31260D & GTAAAGGCTTGTCTGTGTACGGTC & CGTCTCTTCCACTAACCCATCG & SOT16 \\
\hline BnaA04g17910D & CСTCACACTCCACATCATGTTC & TCAAGTGTCTCCTTCCAAGCAG & GSTF9 \\
\hline BnaA07g00460D & GTCGATTTGGTGTGTGATAGGC & CCTCAAGCATATGAGCTTCCAC & SUR1 \\
\hline BnaA03g57920D & TGAGAGAGAGATGGGTCGTGTTC & GCTTCACAATGGACTCACCAAC & IGPS \\
\hline BnaA02g05170D & GCTGTGGATTTAGGACAGATTGAA & CTGGAACAGGCAAGTCAAAGCTC & $\mathrm{AAO} 1$ \\
\hline BnaC04g19270D & GGAGGGATTGAGAATTTGTTGAA & GCGAATCGACATCTCTATGCTC & APR1 \\
\hline BnaC01g00790D & GGTGAAGTGGCAAAGCTGTTG & GATGATGTITTCAGCCATCTGAC & APK2 \\
\hline BnaA03g59800D & GAGGGATTGAGAATCTGTTGAAGC & ATAAGCGAATCCACATCTCTATGC & APR2 \\
\hline BnaA10g11280D & GACGGTTGCTCACGAAACTCTC & ACTAAGCATTGTGTTGCTCTCTCC & CYP81F2 \\
\hline BnaA01g12900D & CATCTGACGACAATATTCCCTGAG & CACATGCTTCAACAAACCATCTCT & GSH1 \\
\hline BnaC02g40130D & ACGTGGTGAATTTCTTGGTCCAC & GTTGACACCCTTCTCCTTGATGAC & TGG2 \\
\hline BnaCnng66020D & ACAAACGGTGGGAGTGGTAA & GTGATCACCGCTCGCATATC & WRKY33 \\
\hline BnaC04g38910D & TGACGGATACAGGTGGAGGAAG & GTCAAGATGTGGTGGAAGTTATCG & WRKY45 \\
\hline BnaA05g28580D & CAGTGATGAACGCAAGATCATC & AAGCCTCCTCTCTCAGACACAAC & SZF1 \\
\hline BnaAnng08970D & TTCTCCAACTCGTCCAGGC & GGCACTTGTCGTAGTTTCCG & GASA1 \\
\hline BnaA09g44500D & CAAGAAGATGGAGAAGTCGGCTAG & CATGAGAAGATTCGAGGTTGGTG & MYB51 \\
\hline BnaC04g38910D & ACGACGGAGCACTACGTCACTATC & ATCACCAGCAGTATAAGCAGAAGC & Bzip44 \\
\hline BnaCnng74400D & GTATTGGATTCAGAAGTGCCTGG & TCTGTGAGATGCACAAATGCTG & TRN1 \\
\hline BnaC01g12390D & CTGATAGTCTCAACACCAAACTTCG & GTCCTTCTGCTACACTCTCAACAGC & HEN1 \\
\hline BnaC03g14590D & ACGAGCTAACCGAAGCTGATG & AAACATTCCAGCACCAAATGTGT & FQR1 \\
\hline BnaA09g05810D & TGCAGATGGTCTTAAGAGCTACCAG & TTCCTTCTTCTCCGACTCATCTCC & CNX1 \\
\hline BnaA09g52180D & ATCCACAAGACATCATGGCTGAG & CTGGTTCATCTTCAATTCCACATTC & CATHB3 \\
\hline BnaC03g30870D & GCCTTTGAAGATGGAGACCTCA & TCGGTGAAGAGCTTCTTGGTG & GSTF2 \\
\hline
\end{tabular}


germinated in pure water in the dark at $20^{\circ} \mathrm{C}$, suggesting that all the seeds were viable. Following secondary dormancy induction via PEG treatment, almost all of the seeds of cultivar $\mathrm{H}$ germinated in pure water (Fig. 1c), while only few of the seeds of cultivar $\mathrm{V}$ germinated (Fig. 1d). To test whether the non-germinated seeds were secondary dormant (viable), secondary dormancy was broken under alternating light/temperature conditions. After dormancy release treatment, all the non-germinated seeds from $\mathrm{H}$ (Fig. 1e) and V (Fig. 1f) germinated, suggesting the non-germinated seeds were viable and secondary dormant. Statistical analyses of the secondary dormancy rate from year 2015 to 2017 revealed that cultivar H exhibited approximately 5\% secondary dormancy potential and cultivar V exhibited approximately 95\% secondary dormancy potential (Table 1).
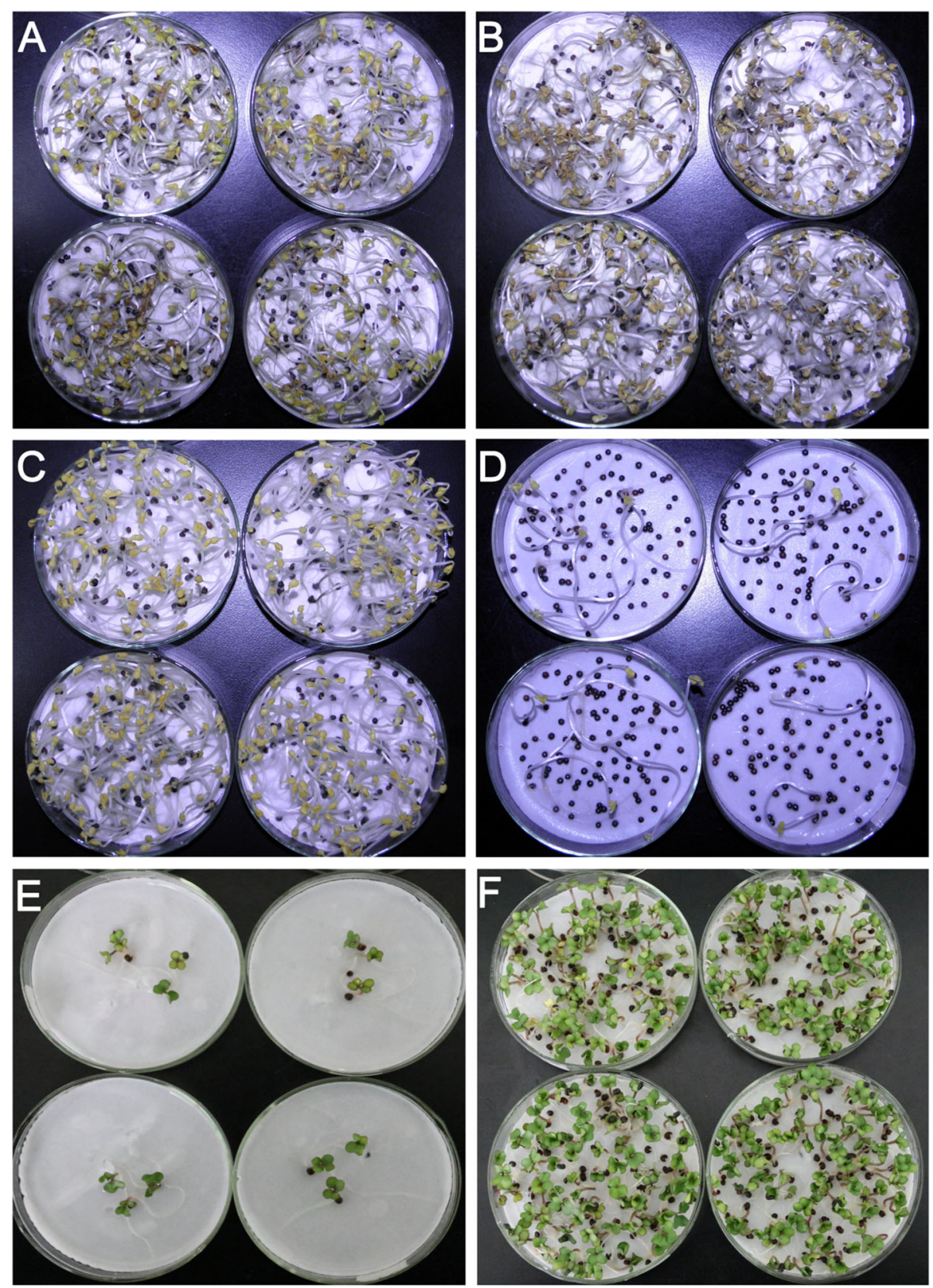

Fig. 1 Characterization of the secondary dormancy phenotypes of cultivars $\mathrm{H}$ and $\mathrm{V}$. (a and $\mathbf{b}$ ) Images of seed germination assay of cultivars $\mathrm{H}$ (a) and $\mathrm{V}(\mathbf{b})$ in pure water in the dark for two weeks, respectively. (c and $\mathbf{d})$ Secondary dormancy phenotypes of cultivars $\mathrm{H}$ (c) and $\mathrm{V}$ (d) following PEG6000 treatment for two weeks and incubation in water for another 8 days in the dark, respectively. (e and $\mathbf{f}$ ) Secondary dormancy release assay of cultivars $\mathrm{H}(\mathbf{e})$ and $V(\mathbf{f})$ following alternating light/temperature conditions, respectively 


\section{Analysis of RNA-seq quality control}

To explore the transcriptomic changes during secondary dormancy induction, the representative cultivars $\mathrm{H}$ and $\mathrm{V}$, which exhibit low (approximately 5\%) and high (approximately 95\%) secondary dormancy potential respectively, were used to perform RNA-seq experiments. The total RNA isolated from mature seeds (named $\mathrm{Hb}$ and $\mathrm{Vb}$ ) and PEG-induced seeds (named $\mathrm{Ha}$ and $\mathrm{Va}$ ) was qualified via Agilent 2100 analyzer and all of the RIN values were $\geq 7.9$ (Additional file 1: Figure S1). A total of 12 cDNA libraries from three independent biological replicates were separately constructed using polyA ${ }^{+}$RNA. The quality of RNAseq experiments relies on the read coverage with respect to the reference genome of oilseed rape and the correlations between biological replicates [29]. The Phred quality score of $>30 \%(\mathrm{Q}>30 \%)$ was $>90 \%$ (Additional file 2 : Table S1), suggesting that the sequencing was of high quality. In addition, approximately $75-82 \%$ of the clean reads were successfully mapped to the reference genome using TopHat 2 , and $66-75 \%$ of the clean reads were blasted to the unique genomic locations (Additional file 2: Table S1). As shown in (Additional file 1: Figure S2), an average of $60 \%$ of the total reads shared $>80 \%$ similarity with the reference genome. Furthermore, the correlations between the repetitions for each sample were $>0.90$ (Additional file 1: Figure S3; Additional file 2: Table S2). These results collectively suggest that the RNA-seq data used in this study were highly reliable.

\section{Characterization of DEGs in secondary dormancy induction}

In $\mathrm{Hb}, \mathrm{Ha}, \mathrm{Vb}$ and $\mathrm{Va}, 57,236,54,273,54,501$ and 53,386 genes were detected, respectively (Fig. 2a). In total, 66, 703 genes accounting for $66.0 \%(66,703 / 101040)$ of the annotated genes in Brassica napus were expressed in the four samples. BLAST queries revealed that 50,589 of the detected 66,703 genes (75.8\%) hit homologues in Arabidopsis, with an $\mathrm{E}<10^{-5}$ for nucleic acids. The detailed analyses of the detected genes including gene expression level, functional annotation, GO and KEGG analysis and MapMan analysis are shown (Additional file 1: Figure S4; Additional file 2: Table S3).

To determine the DEGs involved in secondary dormancy, four comparison settings including $\mathrm{Ha}$ vs $\mathrm{Hb}, \mathrm{Va}$ vs $\mathrm{Vb}, \mathrm{Va}$ vs $\mathrm{Ha}$ and $\mathrm{Hb}$ vs $\mathrm{Vb}$, were analysed with a threshold of $\left|\log _{2}(\mathrm{FC})\right| \geq 1$ and a FDR $\leq 0.001$. Under these criteria, 8553 (4432 up and 4121 down), 7935 (4240 up and 3695 down), 8959 (4357 up and 4602 down) and 6704 (3283 up and 3421 down) genes were significantly differentially expressed in the $\mathrm{Ha}$ vs $\mathrm{Hb}, \mathrm{Va}$ vs $\mathrm{Vb}, \mathrm{Va}$ vs $\mathrm{Ha}$ and $\mathrm{Hb}$ vs $\mathrm{Vb}$ comparisons, respectively (Fig. 2b and c; Additional file 1: Figure S5). Much more DEGs were observed in Va vs Ha among the four comparison settings (Fig. 2b). Moreover, compared with downregulated genes, upregulated genes were identified more often in $\mathrm{Ha}$ vs $\mathrm{Hb}$ and especially in $\mathrm{Va}$ vs $\mathrm{Vb}$ (Fig. 2c). However, more downregulated genes were found both in both $\mathrm{Va}$ vs $\mathrm{Ha}$ and $\mathrm{Hb}$ vs $\mathrm{Vb}$ (Fig. 2c).

\section{Identification of candidate DEGs responsible for secondary dormancy}

To identify the candidate genes associated with secondary dormancy, a Venn diagram containing 15 individual subgroups was constructed in conjunction with the four comparison settings (Fig. 2d). We focused predominately on the DEGs located in the intersection between $\mathrm{Va} v \mathrm{Vb}$ and $\mathrm{Va}$ vs $\mathrm{Ha}$, which was divided into four subgroups (IIV) that contained 428, 513, 430 and 1283 DEGs, respectively (Fig. 2d). Given that the DEGs in subgroups I and II also exhibited significant changes in $\mathrm{Ha}$ vs $\mathrm{Hb}$, which showed non-dormant phenotypes, we collected mainly the inversely expressed genes (i.e., those upregulated in Va vs $\mathrm{Vb}$ but downregulated in $\mathrm{Ha}$ vs $\mathrm{Hb}$ and vice versa). For the DEGs in subgroups III and IV, a more stringent criterion, $\mid \log _{2}$ (Va vs Vb)- $\log _{2}(\mathrm{Ha}$ vs $\mathrm{Hb}) \mid \geq 1$, was used to select the crucial candidate genes involved in secondary dormancy. On the basis of a second round of selection, 25, 44, 114 and 815 DEGs (998 in total) in the four subgroups were isolated, respectively (Additional file 2: Table S4). Among the 998 DEGs, 960 (96.2\%) hit homologues in Arabidopsis and 674 (67.5\%) were named and functionally annotated in Arabidopsis (Additional file 2: Table S4). To explore the expression profiles of the 998 DEGs on the basis of their normalized FPKM values, a heat map was constructed. As shown in Fig. 2e, the heat map clearly shows two clusters for the expression patterns of DEGs in the dormant sample (Va) and the number of bottom clusters (upregulated DEGs) was three times greater than that of the top clusters (downregulated DEGs). Taken together, these results indicated that the majority of candidate DEGs were significantly upregulated in secondary dormancy induction.

\section{Functional characterization of DEGs}

To obtain the functional information of the DEGs, GO subcategory and KEGG pathway analyses were conducted. GO subcategory analysis revealed that "sulfur compound metabolism" and "glucosinolate metabolism" were highly enriched in upregulated DEGs (Table 3; Additional file 2: Table S5). Interestingly, the highly similar representative KEGG pathways "sulfur metabolism", "glucosinolate biosynthesis" and "tryptophan metabolism" were also clearly enriched in upregulated DEGs (Table 3; Additional file 2: Table S6). In addition, the KEGG pathways identified for the DEGs in the first round of selection also suggested that the above three terms were highly enriched (Additional file 1: Figure S6). 
Furthermore, a metabolic map and regulatory map showing an overview of the DEGs between Va and Ha were constructed using MapMan software. With respect to the cellular metabolism visualization, the majority of the DEGs were involved in pathways including lipid metabolism, secondary metabolism, amino biosynthesis and sulfur metabolism (Additional file 1: Figure S7;
Additional file 2: Table S7), which validated the results of the GO and KEGG enrichment analyses. In the regulatory visualization, most of the DEGs were mapped to "transcription factor" "protein modification" "protein degradation" and "phytohormone signalling" (Additional file 1: Figure S7; Additional file 2: Table S7). The detailed information showing all the

\section{A}

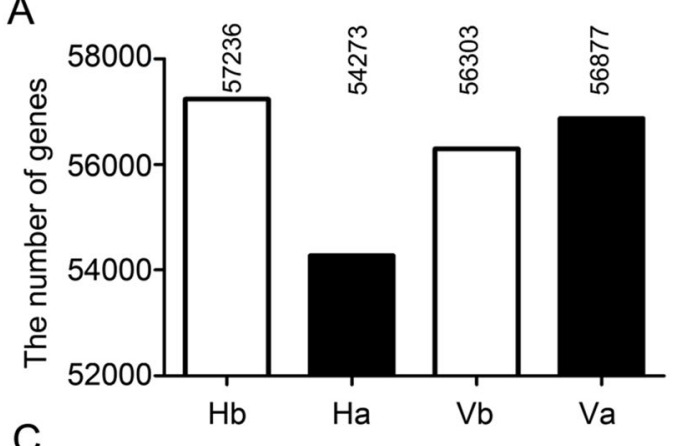

C

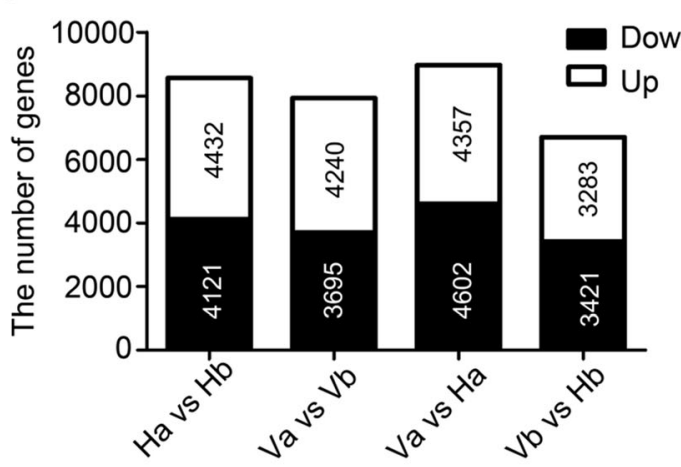

D

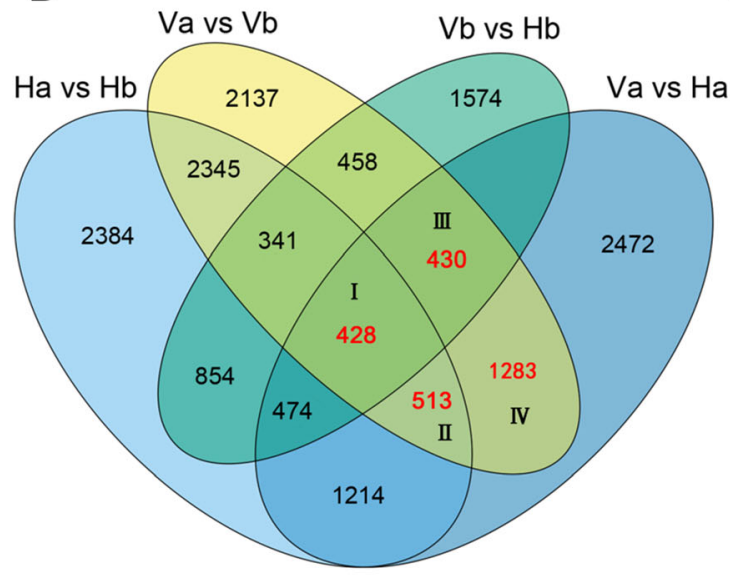

B
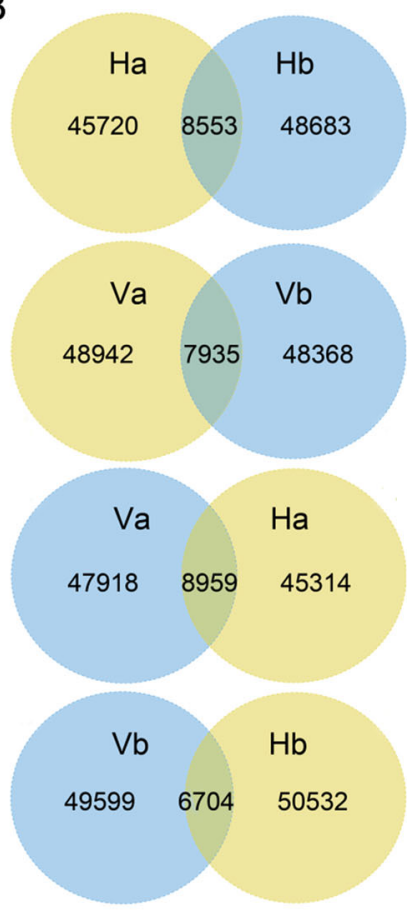

$\mathrm{E}$

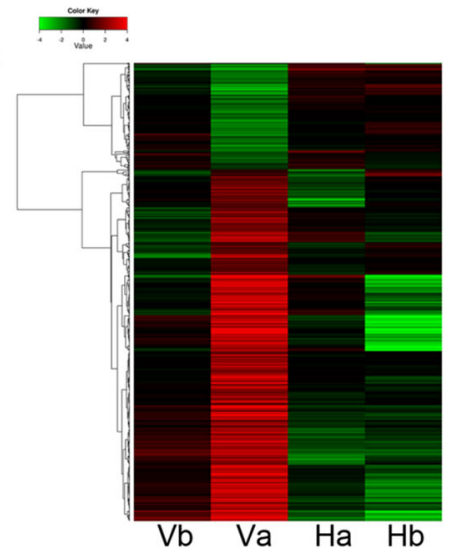

Fig. 2 Identification and expression analysis of the DEGs involved in secondary dormancy. a Comparisons of the expressed gene numbers among the individual samples. The number above the bars indicates the gene number detected in the corresponding sample. $\mathbf{b}$ Venn diagrams showing the numbers of expressed genes and DEGs from the four comparison groups. The numbers in the intersections denote the DEGs in each group. c Comparisons of the number of DEGs among the four comparison groups and the number of up- and downregulated DEGs in the individual comparison groups. The numbers in the open and filled bars indicate the up- and downregulated DEGs numbers, respectively. $\mathbf{d}$ Venn diagram showing the DEGs between two individuals ( $\mathrm{Ha}$ vs $\mathrm{Hb}$, Va vs Vb, Vb vs Hb, Va vs $\mathrm{Ha}$ ) and the candidate DEGs (in red) required for secondary dormancy in the first round of selection. e Hierarchical cluster analysis of 998 DEGs in which normalized FPKM values were used. The red colour denotes upregulated genes, and the green colour indicates downregulated genes, as shown in the above colour bar. The final normalized FPKM values range from -4 to +4 
Table 3 Detailed results of GO subcategory and KEGG pathways analyses for DEGs identified via RNA-seq analysis

\begin{tabular}{|c|c|c|c|}
\hline \multicolumn{2}{|c|}{ Top 10 of detailed GO subcategory analysis } & \multicolumn{2}{|l|}{ Top 10 of KEGG pathways } \\
\hline Downregulated DEGs & Upregulated DEGs & Downregulated DEGs & Upregulated DEGs \\
\hline Protein storage vacuole & Sulfur compound metabolism & Sulfur relay system & Sulfur metabolism \\
\hline Plastid thylakoid membrane & Glycosyl compound metabolism & Fatty acid degradation & Carbon metabolism \\
\hline Oxidoreductase activity & Oxoacid metabolism & Peroxisome & Biosynthesis of amino acids \\
\hline $\begin{array}{l}\text { Intracellular protein transmembrane } \\
\text { import }\end{array}$ & Organic acid metabolism & $\begin{array}{l}\text { Cutin, suberine and wax } \\
\text { biosynthesis }\end{array}$ & Citrate cycle (TCA cycle) \\
\hline Thylakoid membrane & $\begin{array}{l}\text { Carbohydrate derivative } \\
\text { metabolism }\end{array}$ & Carotenoid biosynthesis & 2-Oxocarboxylic acid metabolism \\
\hline Photosynthetic membrane & Secondary metabolic process & Tyrosine metabolism & Cysteine and methionine metabolism \\
\hline Cytoplasm & Response to cadmium ion & alpha-Linolenic acid metabolism & Glucosinolate biosynthesis \\
\hline Seed maturation & S-glycoside metabolic process & $\begin{array}{l}\text { Ascorbate and aldarate } \\
\text { metabolism }\end{array}$ & Tryptophan metabolism \\
\hline Fruit development & Glucosinolate metabolism & Mismatch repair & $\begin{array}{l}\text { Glycine, serine and threonine } \\
\text { metabolism }\end{array}$ \\
\hline Intramolecular lyase activity & Response to metal ion & $\begin{array}{l}\text { Fructose and mannose } \\
\text { metabolism }\end{array}$ & Pyruvate metabolism \\
\hline
\end{tabular}

The enriched pathways in bold are linked with auxin biosynthesis

DEGs corresponding to their MapMan functional categories is listed in Table S7. The combination of the MapMan, KEGG pathway and GO subcategory analyses indicated that the DEGs that were associated with sulfur metabolism, GLS metabolism and phytohormone signalling pathways play dominant roles in secondary dormancy.

\section{Validation of the DEGs involved in indole GLS-linked auxin biosynthesis in secondary dormancy}

The pathways "tryptophan metabolism", "sulfur metabolism", "glucosinolate biosynthesis" and "auxin biosynthesis" are tightly linked (Fig. 3a). In this study, transcriptomic profiles revealed that the expression of the DEGs involved in tryptophan (Trp) biosynthesis in dormant Va seeds was markedly increased (Additional file 2: Table S8). Among the DEGs, BnaA03g01690D, encoding $\alpha$-subunit of anthranilate synthase (ASA1) (Fig. 3a), showed about 20fold and 4-fold upregulation in $\mathrm{Va} v \mathrm{Vb}$ and $\mathrm{Va}$ vs $\mathrm{Ha}$, respectively (Fig. 3b). The expression of BnaA03g57920D, encoding indole-3-glycerol phosphate synthase (IGS) (Fig. 3a), exhibited a 6-fold increase in Va vs $\mathrm{Ha}$ (Fig. 3b). In addition, both Trp synthase $\alpha$ (TSA1) and $\beta$ (TSB1) (Fig. 3a), encoded by BnaC08g25400D and BnaA10g09090D, respectively, were found to be upregulated more than 10 folds in Va vs Ha (Fig. 3b).

In this study, expression of the genes associated with the biosynthesis of sulfur donors was significantly upregulated in $\mathrm{Va}$ vs $\mathrm{Vb}$ and $\mathrm{Va}$ vs $\mathrm{Ha}$ (Additional file 2: Table S8). Indole GLS is biosynthesized from Trp (Fig. 3a). Interestingly, dramatic upregulation was observed only for the majority of indole GLS biosynthesis genes
(Additional file 2: Table S8), while no change was observed for aliphatic or aromatic GLS genes (Additional file 2: Table S9). Furthermore, qRT-PCR validated that the expression of BnaC01g00790D (APK2) was upregulated more than 150 -fold in $\mathrm{Va}$ vs $\mathrm{Vb}$ and $\mathrm{Va}$ vs $\mathrm{Ha}$ (Fig. 3b). In addition, the expression of BnaAO 1g34610D (CYP79B2), BnaA08g04520D (CYP83B1), BnaA07g00460D (SUR1) and Bna04g17910D (GSTF9), encoding the crucial enzymes required for the core structure formation of indole GLS, was upregulated 20fold to 40-fold in Va vs Ha, as shown by qRT-PCR (Fig. $3 \mathrm{~b})$, which matched well with the RNA-seq results. In summary, contrast to that of the aliphatic and aromatic GLS biosynthesis genes, the expression of indole GLS biosynthesis genes was uniquely upregulated in secondary dormancy.

Notably, indole GLS is tightly linked with IAA biosynthesis [38-40]. IAA is biosynthesized from Trpindependent and Trp-dependent pathways [41, 42]. In the present study, expression of the Trp-independent IAA biosynthesis gene INS/TRPA1 showed no change in the dormant seeds (Additional file 2: Table S8). Correspondingly, in the Trp-dependent pathway, the most markedly changed DEG was BnaA02g05170D (AAO1), which was upregulated 150-fold in dormant seeds (Va) compared with the non-dormant seeds ( $\mathrm{Vb}$ and $\mathrm{Ha}$ ). Moreover, expression of the myrosinaseencoding gene BnaC02g40130D (TGG2) which catalyzes indole-GLS to produce IAN for IAA biosynthesis (Fig. 3a) was upregulated more than 5-fold in $\mathrm{Va}$ vs Ha (Fig. 3b). In addition to the strikingly upregulated biosynthesis genes, genes involved in auxin 


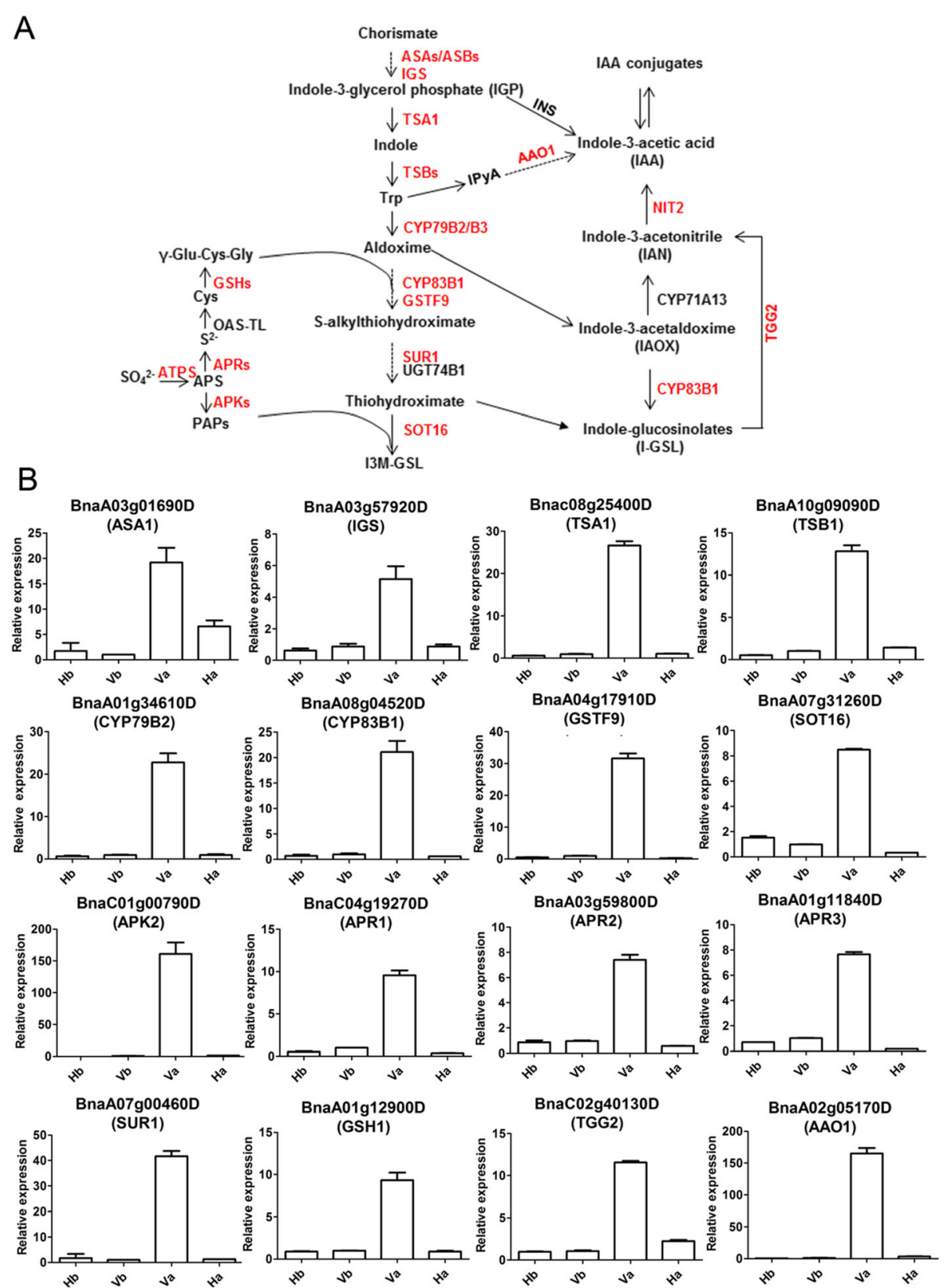

Fig. 3 Validation of the DEGs involved in indole GLS-linked auxin biosynthesis via qRT-PCR. a The enriched metabolic pathway for the significant DEGs involved in secondary dormancy. The DEGs identified in the current study are marked in red. The genes in black were not detected in this study. The solid arrow indicates the direct promotion of the conversion, while the dotted arrow denotes the indirect promotion. $\mathbf{b}$ Verification of the enriched DEGs in (A) via GRT-PCR. Three independent biological replicates were tested and analysed. The relative expression levels of the DEGs were calculated via the $2^{-\triangle \Delta C T}$ method. The corresponding gene name is shown in parentheses, as annotated by BLASTN in Arabidopsis. BnaCAT1 was used as an internal control. Data are mean \pm SD of three independent replicates

signaling transduction including BnaC02g36370D $(A$ FB2), BnaA10g27350D (SSR1), BnaA04g26870D (A BCB4), BnaA07g19210D (ABCB21), BnaC08g19040D (ARF5) and BnaA05g01310D (ARF11) were also significantly changed in Va vs Ha (Table 4), thereby indicating a strong auxin signaling in secondary dormancy.

\section{Determination of the metabolic differences in auxin intermediates}

To investigate whether the activation of indole GLSlinked auxin biosynthesis led to metabolic differences, the concentration of auxin metabolites was determined. The level and distribution of auxin are tightly regulated by its 
synthesis (auxin precursors), inactivation (modified auxin forms) and transport [43]. Free IAA, main auxin precursors (IPyA), auxin substrates (Trp) and inactive IAA conjugates (IAA-aspartate (Asp) and IAA-glutamate (Glu)) were measured in the dry seeds and dormancy-induced seeds (Fig. 4). The level of free IAA was significantly higher in the dormant seeds (Va) than in the nondormant seeds (Ha) (Fig. 4), in agreement with the upregulation of DEGs in the dormant seeds. Surprisingly, a dramatic decrease of free IAA was observed both in $\mathrm{Ha}$ vs $\mathrm{Hb}$ and $\mathrm{Va} \mathrm{vs} \mathrm{Vb}$ (Fig. 4), suggesting that the change was in response to PEG treatment rather than secondary dormancy. The concentration of IPyA, which is considered the predominant precursor for IAA biosynthesis [38], increased in the dormant seeds (Va) (Fig. 4). A negative feedback inhibition of Trp biosynthesis was released when Trp biosynthesis was impaired, leading to an increase in free IAA contents [44]. Similarly, a decrease in Trp concentration was observed in the dormant seeds (Va) (Fig. 4), resulting in the upregulation of Trp biosynthesis genes (Fig. 3b). Moreover, the concentrations of the inactive IAA conjugates (IAA-Asp and IAA-Glu) were much lower in the dormant seeds (Va) than in the non-dormant seeds (Ha and Vb) (Fig. 4). Taken together, these results indicate that indole GLS-linked auxin biosynthesis was enhanced in secondary seed dormancy.

\section{Exogenous IAA application promotes secondary dormancy in rapeseed}

To confirm the effects of auxin on secondary dormancy, exogenous IAA was applied to cultivar H. First, two strategies were applied to treat the low dormancy potential $\mathrm{Hb}$ in the dark: constant treatment with low concentrations of IAA $(0 \mathrm{mg} / \mathrm{L}, 0.1 \mathrm{mg} / \mathrm{L}, 1 \mathrm{mg} / \mathrm{L}, 10 \mathrm{mg} / \mathrm{L}$ and $100 \mathrm{mg} / \mathrm{L})$ (Additional file 1: Figure S8a) and treatment with high concentrations of IAA $(0 \mathrm{mg} / \mathrm{L}, 100 \mathrm{mg} / \mathrm{L}, 500 \mathrm{mg} / \mathrm{L}$, $1000 \mathrm{mg} / \mathrm{L}$ and $2000 \mathrm{mg} / \mathrm{L}$ ) for $24 \mathrm{~h}$ (Additional file 1: Figure S8c). Under constant treatment conditions, the ratio of dormant seeds increased from 20 to $40 \%$ as the exogenous IAA concentration increased (Additional file 1: Figure $\mathrm{S} 8 \mathrm{~b}$ ). When treated with $500 \mathrm{mg} / \mathrm{L}$ IAA for $24 \mathrm{~h}$, the dormant ratio reached up to $80 \%$ (Additional file 1: Figure S8d). The same strategies were applied to treat the low dormancy sample $\mathrm{Ha}$ in the dark. Under conditions of constant application of $100 \mathrm{mg} / \mathrm{L}$ IAA, the dormancy ratio was $40 \%$ greater than that of the control (Fig. 5a and b), and compared with that in the control, the hypocotyl length in the $10 \mathrm{mg} / \mathrm{L}$ and $100 \mathrm{mg} / \mathrm{L}$ IAA treatments significantly decreased (Fig. 5c). Similarly, a 30\% increase in the dormancy ratio and a $38 \%$ decrease in hypocotyl length were observed in response to the $500 \mathrm{mg} / \mathrm{L}$ IAA treatment for $24 \mathrm{~h}$ (Fig. 5d-f). Notably, low concentrations of IAA and the combination of ABA and IAA can't mimic secondary dormancy (Additional file 1: Figure S9), in agreement with ABA treatment [12], but high concentration of IAA increased secondary dormancy ratio and elevated BnaDOG1 expression level (Additional file 1: Figure S9), which is associated with secondary dormancy [4]. Collectively, the experimental results supported the main conclusion that high exogenous auxin enhanced secondary seed dormancy in the above conditions.

\section{Discussion}

A long-standing question is whether secondary dormancy differs mechanistically and physiologically from primary dormancy [45]. Although extensively molecular and genetic analysis have been conducted on primary dormancy, very few researches uncovered the genetic basis underlying secondary dormancy in rapeseed $[7,16$, 46]. In the previous researches, the secondary dormancy

Table 4 List of DEGs involved in auxin transduction identified via RNA-seq analysis

\begin{tabular}{lllll}
\hline Gene ID & Arabidopsis orthologs & Va vs Vb $\left(\log _{2} \mathrm{FC}\right)$ & Va vs Ha $\left(\log _{2} \mathrm{FC}\right)$ & Function description \\
\hline BnaA07g28560D & IAR4 & Up $(1.7)$ & Up (1.9) & IAA conjugate sensitivity \\
$\begin{array}{l}\text { BnaA09g42140D } \\
\text { BnaC08g34560D }\end{array}$ & GH3.3 & Down $(-2.3 /-3.7)$ & Down $(-2.3 /-3.3)$ & IAA-amido synthase activity \\
BnaC02g36370D & AFB2 & Up (1.7) & Up (2.6) & The dominant auxin receptor \\
BnaC01g20800D & RMA2 & Down $(-1.5)$ & Down $(-2.1)$ & Ubiquitinates Auxin binding protein 1 \\
BnaA10g27350D & SSR1 & Down $(-1.7)$ & Down $(-2.6)$ & Auxin polar transport \\
BnaC02g24140D & PILS3 & Up (2.8) & Up (2.9) & Auxin efflux carrier \\
BnaA04g26870D & ABCB4 & Up (5.2) & Up (2.8) & Auxin efflux transporter \\
BnaA07g19210D & ABCB21 & Up (3.9) & Auxin efflux transporter \\
BnaC04g13950D & GSTU5 & Up (2.3) & Up (2.9) & Response to auxin \\
BnaC05g50350D & PATL2 & Up (3.9) & Up (2.1) & Response to auxin \\
BnaC05g23700D & PATL4 & Up (1.2) & Up (1.8) & Response to auxin \\
BnaC08g19040D & ARF5 & Up (2.3) & Up (2.9) & Auxin response factor \\
BnaA05g01310D & ARF11 & Up (3.4) & Up (4.1) & Auxin response factor \\
\hline
\end{tabular}




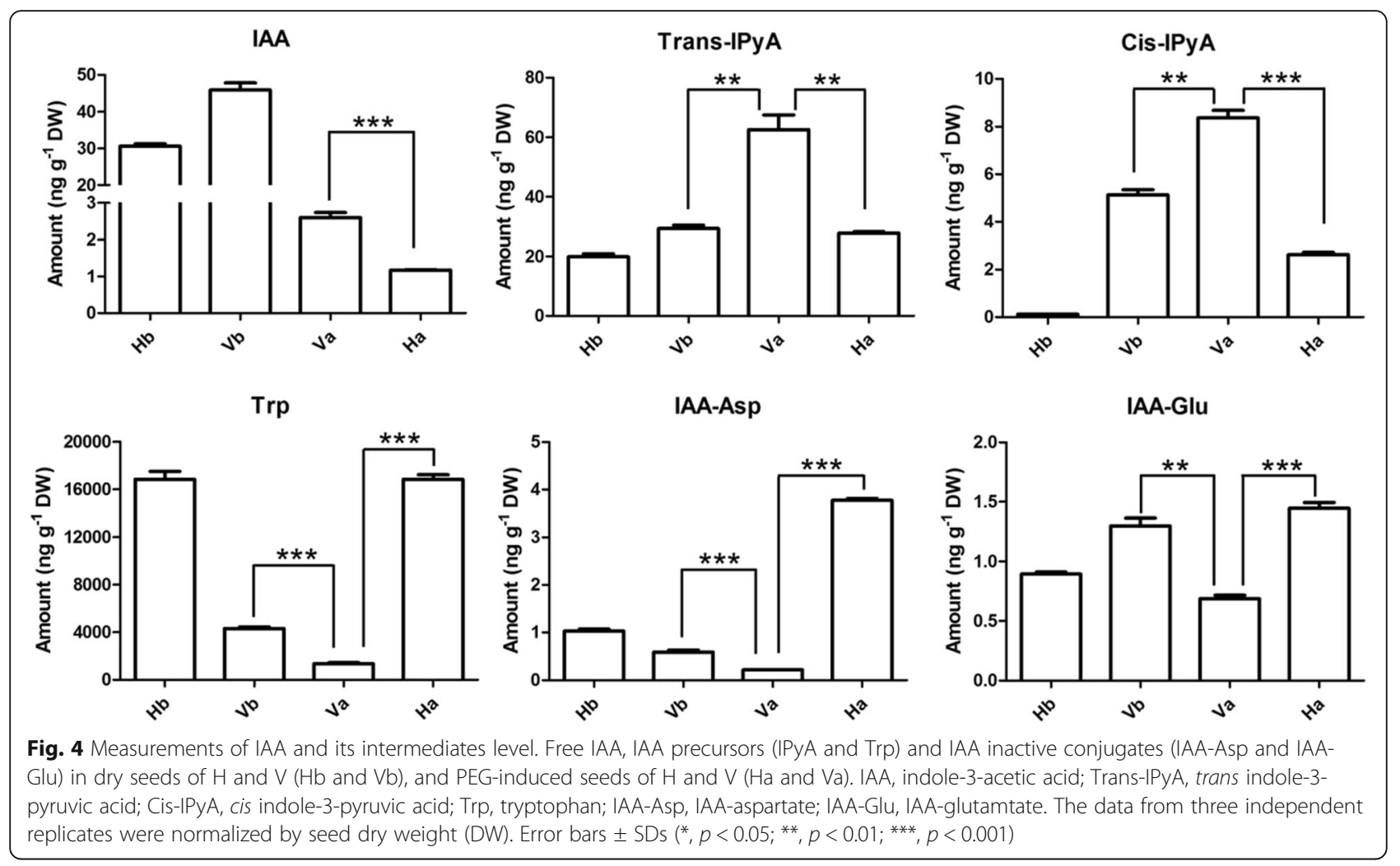

variation in AC Excel (80\%) vs DH12075 (not shown) [7, 46] and in R53 (61.7\%) vs Express 617 (30.4\%) [16] was less significant than that of cultivars V (95\%) vs $\mathrm{H}(5 \%)$ used in this study (Table 1). More importantly, the rapeseed genomic sequence was not released at that time. As such, the cultivars $\mathrm{V}$ and $\mathrm{H}$ served as ideal materials for RNA-seq to explore the candidate DEGs involved in secondary dormancy.

Analyses of all the indexes revealed that the RNA-seq data from three independent biological replicates in this study were of high quality and reliable (Additional file 2: Table S1). Based on a stringent threshold $\left(\mid \log _{2}(\mathrm{FC}) \geq\right.$ 1 and FDR $\leq 0.001$ ), 998 candidate DEGs for secondary dormancy were identified as in $\mathrm{V}$ vs $\mathrm{H}$, which were much more than those isolated in AC Excel vs DH12075 by Arabidopsis AR12K cDNA microarrays [7, 46]. Among the DEGs, those involved in "sulfur metabolism", "tryptophan biosynthesis", "glucosinolate metabolism" (Table 3) and "phytohormone signaling" (Additional file 1: Figure S7) were significantly enriched. GLSs, which are a type of sulfur-rich secondary metabolite composed of a $\beta$-Dglucopyranose residue linked via a sulfur atom to a (Z)-Nhydroximinosulfate ester plus a precursor amino acidderived $\mathrm{R}$ group, are found mainly in Brassicaceae seeds $[47,48]$. On the basis of the precursor amino acid and variable $\mathrm{R}$ group, GLSs can be classified into aliphatic (e.g., alanine (Ala)), indole (Trp) or aromatic (e.g., tyrosine (Tyr)) -GLSs [49]. Notably, different types of GLSs are synthesized by the enzymes of specific genes [48]. In this study, the expression level of specific genes for Trpderived indole GLS biosynthesis were uniquely and -markedly upregulated in the dormant seeds (Fig. 3; Additional file 2: Table S8), while no changes were observed in the expression levels of the aliphatic or aromatic GLS biosynthesis genes (Additional file 2: Table S9). Correspondingly, the expression levels of the transcription factors $B n a A O$ 9g44500D (MYB51), BnaC02g22630D (MYB122) and BnaA05g18020D (MYC2), which positively regulate indole GLS biosynthesis [50], were also upregulated (Additional file 1: Figure S10; Additional file 2: Table S10), while no changes were observed for the expression of Bna Cnng43220D (MYB28) in the dormant seeds (Additional file 2: Table S10), which regulates aliphatic GLS biosynthesis. Notably, phenylpropanoid metabolism showed significant difference between PEG-treated AC Excel and DH12075 seeds [7], which is linked with GLS biosynthesis via aldoxime [23], suggesting that both GLS biosynthesis and related pathways are required for secondary dormancy.

Indole GLS is tightly linked to auxin biosynthesis [48]. Deficiency in GLS metabolism results in severe plant developmental defects that resemble auxin accumulation phenotypes [51-54]. Previous studies have demonstrated that auxin biosynthesis and signaling control primary dormancy in Arabidopsis, although some controversy remains to be solved. Genetic and molecular studies have 

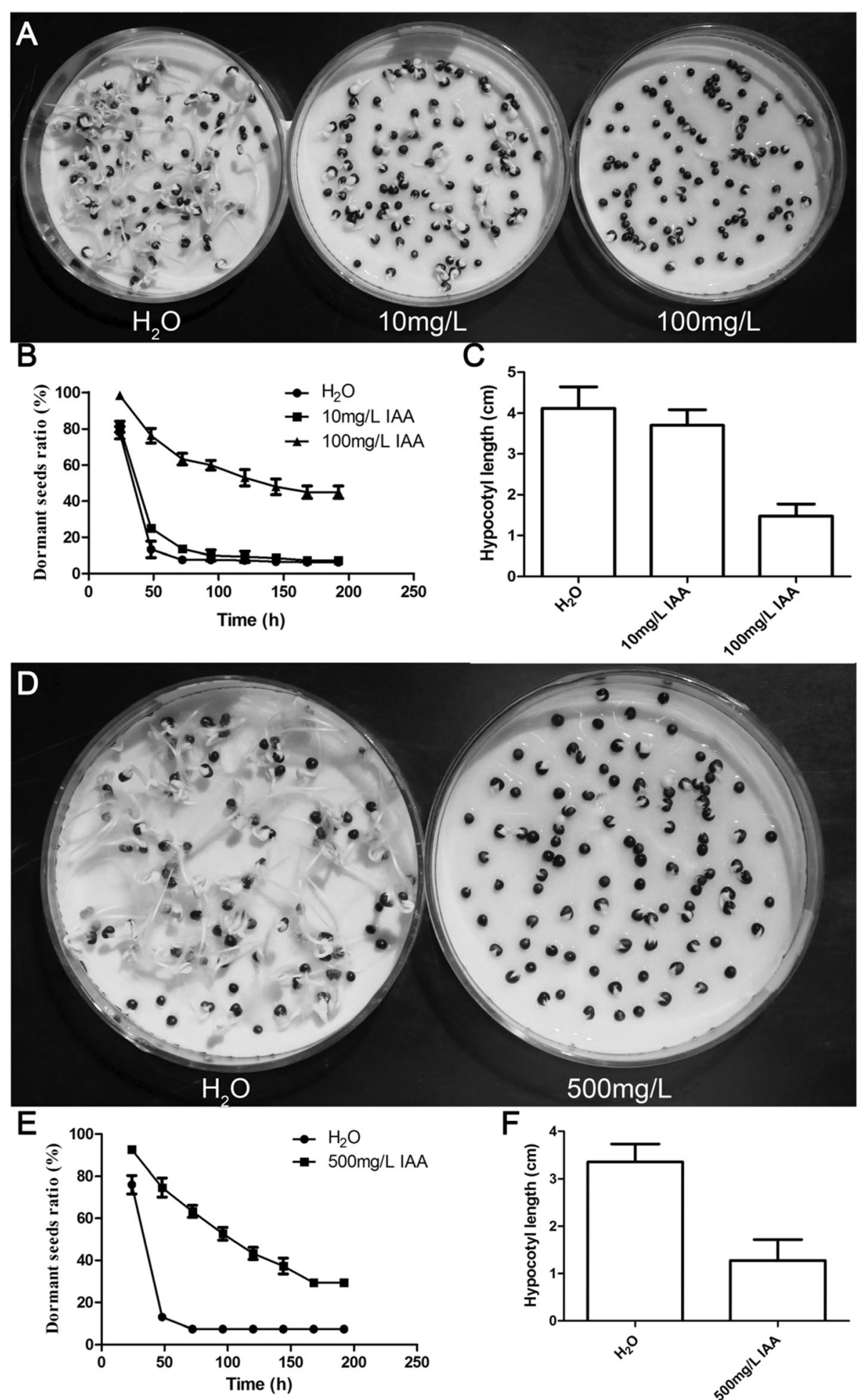

Fig. 5 Effects of exogenous IAA treatment on secondary dormancy. Rape seed dormancy analysis of Ha supplemented with the indicated exogenous IAA solution for 8 days (a-c) and 1 day $(\mathbf{d}-\mathbf{f})$. ( $(\mathbf{a}$ and $\mathbf{d})$ Images of secondary dormancy of Ha supplemented with the indicated exogenous IAA solution. (b and $\mathbf{e})$ Statistics of dormant seed ratios at the indicated times. (c and $\mathbf{f}$ ) Hypocotyl lengths of the geminated seeds grown in the dark for 8 days. The data shown are the means \pm SDs 
Table 5 Summary of DEGs involved in phytohormone biosynthesis and signalling transduction, with the exception of auxin

\begin{tabular}{|c|c|c|c|c|c|}
\hline Hormone & Gene ID & Arabidopsis orthologs & Va vs Vb $\left(\log _{2} \mathrm{FC}\right)$ & Va vs $\mathrm{Ha}\left(\log _{2} \mathrm{FC}\right)$ & Function description \\
\hline \multirow[t]{3}{*}{$A B A$} & BnaA04g16900D & CYP707A2 & Down $(-1.0)$ & Down $(-1.9)$ & ABA degradation \\
\hline & BnaC06g38870D & NCED9 & Up (1.5) & Up (1.2) & ABA biosynthesis \\
\hline & BnaA02g05170D & $\mathrm{AAO} 1$ & Up (5.4) & Up (4.1) & ABA biosynthesis \\
\hline \multirow[t]{3}{*}{ GA } & BnaA09g01090D & GA1 & Down $(-2.0)$ & Down $(-2.6)$ & GA biosynthesis \\
\hline & BnaA10g17240D & RGL3 & Up (2.1) & Up (3.2) & GA negative regulator \\
\hline & BnaAnng08970D & GASA1 & Up (positive) & Up (positive) & GA response factor \\
\hline \multirow[t]{4}{*}{ ACC } & $\begin{array}{l}\text { BnaC09g13570D } \\
\text { BnaA08g14220D }\end{array}$ & $\mathrm{ACO} 2$ & Up (3.0/4.3) & Up (2.0/4.0) & Ethylene biosynthesis \\
\hline & BnaA01g24090D & ETR2 & Up (2.9) & Up (2.4) & Ethylene receptor \\
\hline & $\begin{array}{l}\text { BnaC01g10100D } \\
\text { BnaAnng21280D }\end{array}$ & ERF1 & Up (2.6/4.2) & Up (2.5/2.6) & Response to ethylene \\
\hline & $\begin{array}{l}\text { BnaC09g20090D } \\
\text { BnaA06g35500D }\end{array}$ & ERF2 & $\operatorname{Up}(2.3 / 2.4)$ & Up (2.9/1.3) & Response to ethylene \\
\hline \multirow[t]{4}{*}{$B R$} & BnaC07g47240D & BRI1 & Up (2.4) & Up (2.3) & BR receptor \\
\hline & BnaA08g00410D & BRL1 & Up (2.9) & Up (2.0) & BRI1-like protein \\
\hline & BnaC08g43180D & BSL2 & Up (1.2) & Up (2.2) & BRI1 suppressor \\
\hline & BnaA09g51800D & BSK3 & Up (1.7) & Up (2.1) & BR signaling kinase 3 \\
\hline \multirow[t]{5}{*}{$J A$} & BnaC06g26670D & LOX6 & Up (1.8) & Up (1.2) & JA biosynthesis \\
\hline & BnaA03g56600D & COl1 & Up (1.4) & Up (2.6) & JA receptor \\
\hline & BnaA06g13250D & JAZ1 & Up (positive) & Up (positive) & JA signaling regulator \\
\hline & BnaA10g20060D & JAZ10 & Up (3.4) & Up (positive) & JA signaling regulator \\
\hline & BnaA05g18020D & MYC2 & Up (1.6) & Up (1.6) & JA signaling regulator \\
\hline KAR & BnaA03g53980D & $\mathrm{KAI} 2$ & Down $(-1.7)$ & Down $(-2.5)$ & KAR receptor \\
\hline
\end{tabular}

$A B A$ abscisic acid, $G A$ gibberellic, $A C C$ ethylene, $B R$ brassinolide, $J A$ methyl jasmonate, $K A R$ karrikin

revealed that auxin promotes primary dormancy by recruiting the auxin response factor ARF10 and ARF16 to control the expression of ABI3 in Arabidopsis [23, 24]. In contrast to $A R F 10$ and $A R F 16$, another auxin response factor, $A R F 2$, is involved in seed germination by repressing the ABA signaling pathway [55]. Interestingly, the auxin influx carrier $A U X 1$, which increases IAA levels, was recently reported to positively affect seed germination [26]. Recently, combined transcriptome and translatome analyses have revealed that Trp-dependent auxin biosynthesis inhibits DOG1-dependent primary dormancy in Arabidopsis [23]. In contrast to that conclusion, our data demonstrated that indole GLS-linked auxin biosynthesis increased in secondary seed dormancy (Figs. 3 and 4), and additional exogenous auxin treatment experiments provided evidence that auxin promoted secondary dormancy (Fig. 5), suggesting a discrepancy in primary vs secondary dormancy. As shown by Cadman et al. [56], transcriptomic analysis reveals there are substantial differences in gene expression genome-wide between primary and secondary dormant seeds in Arabidopsis. To our knowledge, this study is the first to provide evidence that auxin, acting as a versatile trigger in plant development, contributes to enhancing secondary dormancy in Brassica napus. However, how the auxin biosynthesis genes are activated remains unknown. In-depth studies on seed treatment applied for different lengths of time could provide insights into this regulation. Moreover, the mechanism by which secondary dormancy is induced by various stressful conditions differs from each other in Arabidopsis [56-58] and in barley [59-61]. It will be very interesting to reveal the specific and general mechanisms underlying secondary dormancy under different conditions in Brassica napus. Notably, the proportion of the explained phenotypic variation of the QTL using the population derived from a cross between AC Excel and DH12075 was somewhat lower, partly due to the less significantly phenotypic variation [16]. Therefore, a population will be constructed using cultivars $\mathrm{V}$ and $\mathrm{H}$ to detect QTL for secondary dormancy in our next work and it will be of great importance for molecular breeding and genomic research.

Extensive interaction between various hormones is required to determine the optimal developmental state for seed germination in various environments [62]. Notably, the exogenous IAA application doesn't completely mimic secondary dormancy because of testa rupture and radical elongation (Fig. 5a and d), which suggested other 
phytohormones or factors are required in dormancy induction. In agreement, the MapMan overview of the regulation indicated that not only auxin but also other phytohormones are involved in secondary dormancy in Brassica napus (Table 5; Additional file 1: Figure S7). ABA level was significantly elevated in AC Excel secondary dormant seeds [7] and two out of four QTL confidence intervals for secondary dormancy partly overlap with those for $A B A$ contents [16], suggesting $A B A$ is a key factor in secondary dormncy. In agreement, the expression of the genes involved in ABA metabolism were significantly up-regulated in Va (Table 5). GA, which antagonistically functions with $\mathrm{ABA}$, were down-regulated in Va (Table 5), further suggesting that the transcriptomic analysis was highly reliable. Surprisingly, another phytohormone, ethylene, which promotes seed germination, was found to be upregulated in dormant seeds (Table 5). Notably, a line of evidence has proved that auxin enhances ethylene production [63], which in turn specifically activates the indole GLS biosynthesis gene [64], suggesting an increase in ethylene may be a secondary reaction in response to auxin in secondary dormancy. Moreover, accumulating evidence has indicated that both brassinosteroid (BR) and auxin pathways synergistically control several auxin-responsive genes [65]. In this study, BR signalling components were upregulated in the dormant seeds. Although the role of jasmonic acid (JA) in seed dormancy is contradictory, JA specifically induces the expression of indole GLS biosynthesis genes [64], suggesting a synergistic effect on secondary dormancy with auxin. In addition to the aforementioned phytohormones, karrikins (KAR), the low-molecularweight organic chemicals derived from the combustion of plant materials, have been shown to promote seed germination via elevated expression of a GA biosynthesis gene [66]. In the secondary dormant seeds, the expression of $K A I 2$, the karrikin receptor, was significantly downregulated (Table 5). Together, all of these results suggest that the cross-talk between auxin and other phytohormones sophisticatedly regulates secondary seed dormancy in Brassica napus.

In addition, the MapMan overview of cellular metabolism revealed that the DEGs are involved mainly in cell walls, lipid metabolism, secondary metabolism (Additional file 1: Figure S7a) and the regulation indicated the DEGs are enriched in transcription factors (Additional file 1: Figure S7b). Expression of cell wallrelated genes, including BnaA10g24090D (PGIP1), BnaA10g24090D (PGIP1) [67] and BnaC08g06930D (STOP1) [68]; lipid metabolism genes, including BnaA06g24040D (ACX2) and BnaC03g49190D (ACX2) [69]; and secondary metabolism-related genes, including BnaA08g21730D (CAT3), BnaC08g19360D (CAT3) $[69,70]$ and BnaA03g52000D (APX6) [71], all of which were proved to be implicated in dormancy in Arabidopsis, exhibited significant changes in both Va vs $\mathrm{Vb}$ and $\mathrm{Va}$ vs $\mathrm{Ha}$ (Additional file 2: Table S3). In agreement, some of the DEGs involved in the pathways mentioned above was also identified in AC Excel vs DH12075 [7]. Several DEGs, including transcription factors, epigenetic modifiers and secondary metabolism genes were randomly selected for validation by qRT-PCR

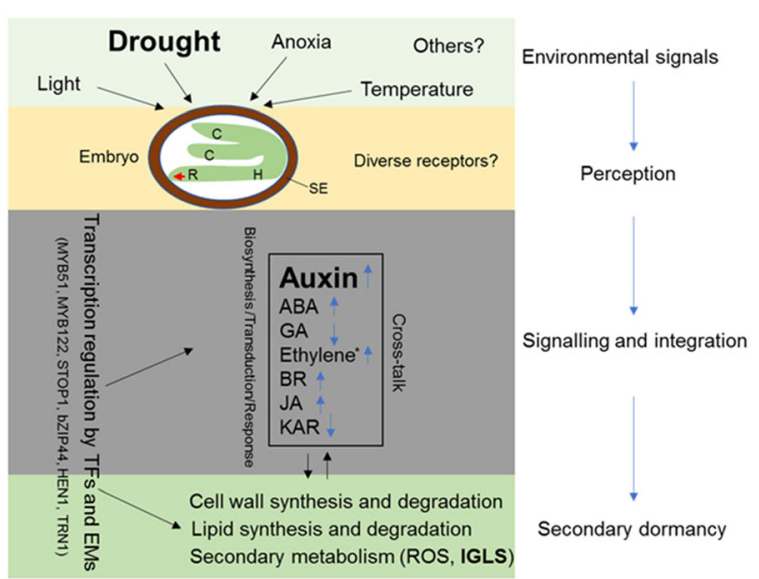

Fig. 6 Schematic diagram for the mechanism underlying secondary seed dormancy induced by drought based on the DEGs in oilseed rape. Non-dormant rape seeds can be induced into secondary dormancy by adverse conditions (light, anoxia, temperature and drought) to keep alive to meet with the proper seasons for germination. The environmental signals are perceived by receptors in the embryo, possibly by diverse receptors. The signals are then transduced and eventually alter phytohormone signalling, including biosynthesis, transduction and responses. The phytohormone signals are integrated to regulate the expression of cell wall-, lipid- and secondary metabolism-related genes by transcription factors and epigenetic modifiers to induce and maintain secondary seed dormancy. PEG6000 is used as a proxy for drought in this study. C, cotyledon; H, hypocotyl; R, root; SE, seed envelope; ABA, abscisic acid; GA, gibberellic; BR, brassinolide; JA, methyl jasmonate; KAR, karrikin; TFs, transcription factors; Ems, epigenetic modifiers; IGLS, indole GLS 
(Additional file 1: Figure S10), and the results generally matched well with the RNA-seq analysis.

\section{Conclusions}

In this study, differentially expressed genes underlying secondary dormancy are explored by comparative transcriptome analysis using high secondary dormancy cultivar $\mathrm{V}$ and low secondary dormancy cultivar $\mathrm{H}$. Our results showed that indole GLS-linked auxin biosynthesis genes are enriched and up-regulated in secondary dormant seeds (Va) compared with non-dormant ones ( $\mathrm{Ha})$ and subsequently confirmed by altered concentrations of indole-3-acetic acid (IAA), IAA conjugates and precursors. Based on our findings in this work, as well as those reported previously, a putative working model with respect to secondary dormancy induction was proposed (Fig. 6). In this model, stressful environmental cues are perceived by receptors in embryo and the signals are then transduced to alter various phytohormones biosynthesis and signaling, especially auxin biosynthesis genes are highly elevated (Fig. 3) in this study. The crosstalk among the phytohormone signaling is eventually integrated to regulate the cell wall-, lipid- and secondary metabolism-related genes by transcription factors and epigenetic modifiers (Additional file 1: Figure S10) to induce the secondary seed dormancy. This study reveals the important role of auxin in secondary dormancy in rapeseed, which will provide valuable information to decrease the soil seed bank.

\section{Additional files}

Additional file 1: Figure S1. RNA quality was qualified by agarose gel electrophoresis (A) and RIN value (B). Figure S2. Distribution of read coverage against the reference genome of oilseed rape. Figure $\mathbf{S 3}$. Correlation analyses for each sample among three repetitions. Figure $\mathbf{S 4 .}$ Overview of the expression of genes in $\mathrm{Va}, \mathrm{Vb}, \mathrm{Ha}$ and $\mathrm{Hb}$ implicated in cellular metabolism (A) and regulation (B) pathways. Figure S5. Volcano plots for the total expressed genes among $\mathrm{Ha}$ vs $\mathrm{Hb}$, Va vs $\mathrm{Ha}$, Va vs $\mathrm{Vb}$ and $\mathrm{Vb}$ vs $\mathrm{Hb}$. Figure S6. KEGG enrichment analysis corresponding to the secondary dormancy candidate DEGs. Figure S7. The MapMan overview of the cellular metabolism process (A) and regulation pathway (B) in which 998 DEGs are involved in. Figure S8. Germination assay with exogenous IAA application to $\mathrm{Hb}$. Figure S9. Effects of ABA and IAA on secondary dormancy. Figure S10. Validation of 12 randomly selected DEGs via qRT-PCR. (DOCX $4606 \mathrm{~kb}$ )

Additional file 2: Table S1. Statistics of the RNA-seq results for the secondary dormancy of 12 samples. Table S2. Correlation analyses for each sample among three repetitions. Table S3. Detailed information of RNA-seq analysis. Table S4. DEGs isolated by the second round of selection based on the threshold $\mid \log 2(\mathrm{Va}$ vs $\mathrm{Vb})-\log 2(\mathrm{Ha}$ vs $\mathrm{Hb}) \mid \geq 1$. Table S5. Detailed GO enrichment analysis of the DEGs. Table S6. Detailed KEGG enrichment analysis of the DEGs. Table S7. Detailed list of all the DEGs corresponding to MapMan functional categories. Table S8. Detailed list of the DEGs involved in indole GLS-linked auxin biosynthesis. Table S9. List of genes involved in aliphatic and aromatic GLSs. Table S10. Differentially expressed transcription factors identified via RNA-seq analysis. Table S11. Differentially expressed epigenetic modifiers identified via RNA-seq analysis. Table $\mathbf{S 1 2}$. Seed quality differences in cultivars $\mathrm{H}$ and V. (XLSX $31002 \mathrm{~kb})$

\section{Abbreviations}

ABA: Abscisic acid; GA: Gibberellic; GLS: Glucosinolate; IAA: Indole-3-acetic acid; IAA-Asp: IAA-aspartate; IAA-Glu: IAA-glutamate; IpyA: Indole-3-pyruvic acid; PEG: Polyethylene glycol; Trp: Tryptophan

\section{Acknowledgements}

None.

\section{Authors' contributions}

$\mathrm{XZ}$ conceived and designed the experiment; JC measured the content of auxin intermediates and analysed the data; $L L$ and $F L$ performed the rest experiments and analysed the data; XY, WF, TT, GC and QG assisted with the experiments; $X Z$ and $L L$ wrote and revised the manuscript. All authors read and approved the final manuscript.

\section{Funding}

This work was supported by the National Natural Science Foundation of China (Grant No. 31470572) and Qing Lan Project of Jiangsu Province (2016). The funders had no role in study design, data collection, data analysis, data interpretation, the writing of the manuscript or decision to publish.

\section{Availability of data and materials}

The dataset generated and analyzed during the study are available from the corresponding author on reasonable request.

Ethics approval and consent to participate

Not applicable.

\section{Consent for publication}

Not applicable.

\section{Competing interests}

These authors declared that they have no competing interests.

\section{Author details}

1Jiangsu Key Laboratory for Eco-agriculture Biotechnology around Hongze Lake, Huaiyin Normal University, Huai'an 223300, China. ${ }^{2}$ Jiangsu

Collaborative Innovation Center of Regional Modern Agriculture and

Environment Protection, Huaiyin Normal University, Huai'an 223300, China.

${ }^{3}$ School of Food Science and Engineering, Yangzhou University, Yangzhou 225127, China. ${ }^{4}$ National Centre for Plant Gene Research (Beijing), Institute of Genetics and Developmental Biology, Chinese Academy of Sciences, Beijing, China.

Received: 19 February 2019 Accepted: 3 June 2019

Published online: 18 June 2019

\section{References}

1. Finkelstein R, Reeves W, Ariizumi T, Steber C. Molecular aspects of seed dormancy. Annu Rev Plant Biol. 2008;59:387-415.

2. Penfield S, MacGregor DR. Effects of environmental variation during seed production on seed dormancy and germination. J Exp Bot. 2017;68:819-25.

3. Chahtane H, Kim W, Lopez-Molina L. Primary seed dormancy: a temporally multilayered riddle waiting to be unlocked. J Exp Bot. 2017;68:857-69.

4. Née G, Obeng-Hinneh E, Sarvari P, Nakabayashi K, Soppe W. Secondary dormancy in Brassica napus is correlated with enhanced BnaDOG1 transcript levels. Seed Sci Res. 2015;(2):1-9.

5. Pekrun C, Lutman PJW, Baeumer K. Germination behaviour of dormant oilseed rape seeds in relation to temperature. Weed Res. 1997;37:419-31.

6. Finch-Savage WE, Leubner-Metzger G. Seed dormancy and the control of germination. New Phytol. 2006;171:501-23.

7. Fei H, Ferhatoglu Y, Tsang E, Huang D, Cutler AJ. Metabolic and hormonal processes associated with the induction of secondary dormancy in Brassica napus seeds. Botany. 2009;87:585-96.

8. Momoh EJJ, Zhou WJ, Kristiansson B. Variation in the development of secondary dormancy in oilseed rape genotypes under conditions of stress. Weed Res. 2002;42:446-55.

9. Hewitt JDJ, Lutman PJW. Cultural control of volunteer oilseed rape (Brassica napus). J Agr Sci. 1998;130:155-63.

10. Lutman PJW, Berry K, Payne RW, Simpson E, Sweet JB, Champion GT, May MJ, Wightman P, Walker K, Lainsbury M. Persistence of seeds from crops of 
conventional and herbicide tolerant oilseed rape (Brassica napus). Proc Biol Sci. 2005:272:1909-15.

11. Gulden RH, Thomas AG, Shirtliffe SJ. Relative contribution of genotype, seed size and environment to secondary seed dormancy potential in Canadian spring oilseed rape (Brassica napus). Weed Res. 2004;44:97-106.

12. Gulden RH, Chiwocha S, Abrams S, McGregor I, Kermode A, Shirtliffe S. Response to abscisic acid application and hormone profiles in spring Brassica napus seed in relation to secondary dormancy. Can J Bot. 2004;82:1618-24.

13. Roller A, Beismann $H$, Albrecht $H$. Persistence of genetically modified, herbicide-tolerant oilseed rape - first observations under practically relevant conditions in South Germany. J Plant Dis Protect. 2002;18:255-60.

14. Liu F, Zhao X, Zhang L, Tang T, Lu C, Chen G, Wang X, Bu C, Zhao X. RNAseq profiling the transcriptome of secondary seed dormancy in canola (Brassica napus L.). Chin Sci Bull. 2014:4341-51.

15. Schatzki J, Allam M, Klöppel C, Nagel M, Börner A, Möllers C. Genetic variation for secondary seed dormancy and seed longevity in a set of blackseeded European winter oilseed rape cultivars. Plant Breed. 2013;132:174-9.

16. Schatzki J, Schoo B, Ecke W, Herrfurth C, Feussner I, Becker HC, Mollers C. Mapping of QTL for seed dormancy in a winter oilseed rape doubled haploid population. Theor Appl Genet. 2013;126:2405-15.

17. Gruber S, Pekrun C, Claupein W. Seed persistence of oilseed rape (Brassica napus): variation in transgenic and conventionally bred cultivars. J Agric Sci. 2004;142:29-40.

18. Pekrun C, Lutman PJW, Baeumer K. Induction of secondary dormancy in rape seeds (Brassica napus L.) by prolonged imbibition under conditions of water stress or oxygen deficiency in darkness. Eur J Agr. 1997;6:245-55.

19. Bassel GW. To grow or not to grow? Trends Plant Sci. 2016;21:498-505.

20. Graeber K, Nakabayashi K, Miatton E, Leubner-Metzger G, Soppe WJ. Molecular mechanisms of seed dormancy. Plant Cell Environ. 2012;35: 1769-86.

21. Nonogaki $\mathrm{H}$. Seed dormancy and germination-emerging mechanisms and new hypotheses. Front Plant Sci. 2014;5:233.

22. Shu K, Liu XD, Xie Q, He ZH. Two faces of one seed: hormonal regulation of dormancy and germination. Mol Plant. 2016;9:34-45.

23. Bai B, Novak O, Ljung K, Hanson J, Bentsink L. Combined transcriptome and translatome analyses reveal a role for tryptophan-dependent auxin biosynthesis in the control of DOG1-dependent seed dormancy. New Phytol. 2018;217:1077-85.

24. Liu PP, Montgomery TA, Fahlgren N, Kasschau KD, Nonogaki H, Carrington JC. Repression of AUXIN RESPONSE FACTOR10 by microRNA160 is critical for seed germination and post-germination stages. Plant J. 2007;52:133-46.

25. Liu X, Zhang H, Zhao Y, Feng Z, Li Q, Yang HQ, Luan S, Li J, He ZH. Auxin controls seed dormancy through stimulation of abscisic acid signaling by inducing ARF-mediated AB/3 activation in Arabidopsis. Proc Natl Acad Sci. 2013;110:15485-90.

26. Wang Z, Chen F, Li X, Cao H, Ding M, Zhang C, Zuo J, Xu C, Xu J, Deng X, et al. Arabidopsis seed germination speed is controlled by SNL histone deacetylase-binding factor-mediated regulation of AUX1. Nat Commun. 2016;7:13412.

27. Yong HY, Zou Z, Kok EP, Kwan BH, Chow K, Nasu S, Nanzyo M, Kitashiba H, Nishio T. Comparative transcriptome analysis of leaves and roots in response to sudden increase in salinity in Brassica napus by RNA-seq. Biomed Res Int. 2014;19:467395

28. Dun X, Tao Z, Wang J, Wang X, Liu G, Wang H. Comparative transcriptome analysis of primary roots of Brassica napus seedlings with extremely different primary root lengths using RNA sequencing. Front Plant Sci. 2016;7:1238.

29. Zhou L, Yan T, Chen X, Li Z, Wu D, Hua S, Jiang L. Effect of high night temperature on storage lipids and transcriptome changes in developing seeds of oilseed rape. J Exp Bot. 2018;69:1721-33.

30. Patel RK, Jain M. NGS QC toolkit: a toolkit for quality control of next generation sequencing data. PLoS One. 2012;7:e30619.

31. Kim D, Langmead B, Salzberg SL. HISAT: a fast spliced aligner with low memory requirements. Nat Methods. 2015;12:357-60.

32. Mortazavi A, Williams BA, McCue K, Schaeffer L, Wold B. Mapping and quantifying mammalian transcriptomes by RNA-Seq. Nat Methods. 2008; 5:621-8.

33. Trapnell C, Roberts A, Goff L, Pertea G, Kim D, Kelley DR, Pimentel H, Salzberg SL, Rinn JL, Pachter L. Differential gene and transcript expression analysis of RNA-seq experiments with TopHat and cufflinks. Nat Protoc. 2012;7:562-78
34. Trapnell C, Hendrickson DG, Sauvageau M, Goff L, Rinn JL, Pachter L. Differential analysis of gene regulation at transcript resolution with RNA-seq. Nat Biotechnol. 2013;31:46-53.

35. Livak KJ, Schmittgen TD. Analysis of relative gene expression data using real-time quantitative PCR and the $2^{-\Delta \Delta C T}$ method. Methods. 2001;25:402-8.

36. Fu J, Chu J, Sun X, Wang J, Yan C. Simple, rapid, and simultaneous assay of multiple carboxyl containing phytohormones in wounded tomatoes by UPLC-MS/MS using single SPE purification and isotope dilution. Anal Sci. 2012:28:1081-7.

37. Mashiguchi K, Tanaka K, Sakai T, Sugawara S, Kawaide H, Natsume M, Hanada A, Yaeno T, Shirasu K, Yao H, et al. The main auxin biosynthesis pathway in Arabidopsis. Proc Natl Acad Sci. 2011;108:18512-7.

38. Ljung K. Auxin metabolism and homeostasis during plant development. Development. 2013;140:943-50.

39. Sugawara S, Hishiyama S, Jikumaru Y, Hanada A, Nishimura T, Koshiba T, Zhao Y, Kamiya Y, Kasahara H. Biochemical analyses of indole-3acetaldoxime-dependent auxin biosynthesis in Arabidopsis. Proc Natl Acad Sci. 2009;106:5430-5.

40. Zhao Y, Hull AK, Gupta NR, Goss KA, Alonso J, Ecker JR, Normanly J, Chory J, Celenza JL. Trp-dependent auxin biosynthesis in Arabidopsis: involvement of cytochrome P450s CYP79B2 and CYP79B3. Genes Dev. 2002;16:3100-12.

41. Normanly J. Approaching cellular and molecular resolution of auxin biosynthesis and metabolism. Cold Spring Harb Perspect Biol. 2010;2:a001594.

42. Woodward AW, Bartel B. Auxin: regulation, action, and interaction. Ann Bot. 2005;95:707-35.

43. Korasick DA, Enders TA, Strader LC. Auxin biosynthesis and storage forms. J Exp Bot. 2013;64:2541-55.

44. Wang B, Chu J, Yu T, Xu Q, Sun X, Yuan J, Xiong G, Wang G, Wang Y, Li J. Tryptophan-independent auxin biosynthesis contributes to early embryogenesis in Arabidopsis. Proc Natl Acad Sci. 2015;112:4821-6.

45. Hilhorst HWM. Definitions and hypotheses of seed dormancy. In Annual plant reviews. Volume 27: seed development, dormancy and germination. doi:https://doi.org/10.1002/9780470988848.ch3.

46. Fei $\mathrm{H}$, Tsang E, Cutler AJ. Gene expression during seed maturation in Brassica napus in relation to the induction of secondary dormancy. Genomics. 2007:89:419-28.

47. Agerbirk N, Olsen CE. Glucosinolate structures in evolution. Phytochemistry. 2012;77:16-45.

48. Malka SK, Cheng Y. Possible interactions between the biosynthetic pathways of indole glucosinolate and auxin. Front Plant Sci. 2017;8:2131.

49. Fahey JW, Zalcmann AT, Talalay P. The chemical diversity and distribution of glucosinolates and isothiocyanates among plants. Phytochemistry. 2001;56:5-51.

50. Frerigmann $H$, Glawischnig E, Gigolashvili T. The role of MYB34, MYB51 and MYB122 in the regulation of camalexin biosynthesis in Arabidopsis thaliana. Front Plant Sci. 2015;6:654.

51. Boerjan W, Cervera MT, Delarue M, Beeckman T, Dewitte W, Bellini C, Caboche M, Van Onckelen H, Van Montagu M, Inze D. Superroot, a recessive mutation in Arabidopsis, confers auxin overproduction. Plant Cell. 1995;7:1405-19.

52. Mikkelsen MD, Hansen $\mathrm{CH}$, Wittstock U, Halkier BA. Cytochrome P450 CYP79B2 from Arabidopsis catalyzes the conversion of tryptophan to indole3-acetaldoxime, a precursor of indole glucosinolates and indole-3-acetic acid. J Biol Chem. 2000;275:33712-7.

53. Mikkelsen MD, Naur P, Halkier BA. Arabidopsis mutants in the C-S lyase of glucosinolate biosynthesis establish a critical role for indole-3-acetaldoxime in auxin homeostasis. Plant J. 2004;37:770-7.

54. Sanchez-Parra B, Frerigmann H, Alonso MM, Loba VC, Jost R, Hentrich M, Pollmann S. Characterization of four bifunctional plant IAM/PAMAmidohydrolases capable of contributing to auxin biosynthesis. Plants (Basel). 2014;3:324-47.

55. Wang L, Hua D, He J, Duan Y, Chen Z, Hong X, Gong Z. Auxin response Factor2 (ARF2) and its regulated homeodomain gene HB33 mediate abscisic acid response in Arabidopsis. PLoS Genet. 2011;7:e1002172.

56. Cadman CS, Toorop PE, Hilhorst HW, Finch-Savage WE. Gene expression profiles of Arabidopsis cvi seeds during dormancy cycling indicate a common underlying dormancy control mechanism. Plant J. 2006;46:805-22.

57. Ibarra SE, Tognacca RS, Dave A, Graham IA, Sanchez RA, Botto JF. Molecular mechanisms underlying the entrance in secondary dormancy of Arabidopsis seeds. Plant Cell Environ. 2016;39:213-21.

58. Lim S, Park J, Lee N, Jeong J, Toh S, Watanabe A, Kim J, Kang H, Kim $\mathrm{DH}$, Kawakami N, et al. ABA-insensitive3, ABA-insensitive5, and DELLAs 
interact to activate the expression of SOMNUS and other hightemperature-inducible genes in imbibed seeds in Arabidopsis. Plant Cell. 2013;25:4863-78.

59. Hoang HH, Sechet J, Bailly C, Leymarie J, Corbineau F. Inhibition of germination of dormant barley (Hordeum vulgare L.) grains by blue light as related to oxygen and hormonal regulation. Plant Cell Environ. 2014;37: 1393-403.

60. Hoang HH, Bailly C, Corbineau F, Leymarie J. Induction of secondary dormancy by hypoxia in barley grains and its hormonal regulation. J Exp Bot. 2013;64:2017-25.

61. Leymarie J, Robayo-Romero ME, Gendreau E, Benech-Arnold RL, Corbineau $F$. Involvement of ABA in induction of secondary dormancy in barley (Hordeum vulgare L.) seeds. Plant Cell Physiol. 2008;49:1830-8.

62. Toh S, Kamiya Y, Kawakami N, Nambara E, McCourt P, Tsuchiya Y. Thermoinhibition uncovers a role for strigolactones in Arabidopsis seed germination. Plant Cell Physiol. 2012:53:107-17.

63. Arteca RN, Arteca JM. Effects of brassinosteroid, auxin, and cytokinin on ethylene production in Arabidopsis thaliana plants. J Exp Bot. 2008;59: 3019-26.

64. Mikkelsen MD, Petersen BL, Glawischnig E, Jensen AB, Andreasson E, Halkier BA. Modulation of CYP79 genes and glucosinolate profiles in Arabidopsis by defense signaling pathways. Plant Physiol. 2003:131:298-308.

65. Tian H, LV B, Ding T, Bai M, Ding Z. Auxin-BR interaction regulates plant growth and development. Front Plant Sci. 2017:8:2256.

66. Nelson DC, Flematti GR, Riseborough JA, Ghisalberti EL, Dixon KW, Smith SM. Karrikins enhance light responses during germination and seedling development in Arabidopsis thaliana. Proc Natl Acad Sci. 2010;107:7095-100.

67. Kanai M, Nishimura M, Hayashi M. A peroxisomal ABC transporter promotes seed germination by inducing pectin degradation under the control of ABI5. Plant J. 2010;62:936-47.

68. Kobayashi Y, Ohyama Y, Ito H, luchi S, Fujita M, Zhao CR, Tanveer T, Ganesan M, Kobayashi M, Koyama H. STOP2 activates transcription of several genes for Al- and low pH-tolerance that are regulated by STOP1 in Arabidopsis. Mol Plant. 2014;7:311-22.

69. Liu WC, Han TT, Yuan HM, Yu ZD, Zhang LY, Zhang BL, Zhai S, Zheng SQ, LU YT. CATALASE2 functions for seedling postgerminative growth by scavenging $\mathrm{H}_{2} \mathrm{O}_{2}$ and stimulating ACX2/3 activity in Arabidopsis. Plant Cell Environ. 2017:40:2720-8.

70. Queval G, Issakidis-Bourguet E, Hoeberichts FA, Vandorpe M, Gakiere B, Vanacker H, Miginiac-Maslow M, Van Breusegem F, Noctor G. Conditional oxidative stress responses in the Arabidopsis photorespiratory mutant cat2 demonstrate that redox state is a key modulator of daylength-dependent gene expression, and define photoperiod as a crucial factor in the regulation of $\mathrm{H}_{2} \mathrm{O}_{2}$-induced cell death. Plant J. 2007;52:640-57.

71. Chen C, Letnik I, Hacham Y, Dobrev P, Ben-Daniel BH, Vankova R, Amir R Miller G. ASCORBATE PEROXIDASE6 protects Arabidopsis desiccating and germinating seeds from stress and mediates cross talk between reactive oxygen species, abscisic acid, and auxin. Plant Physiol. 2014;166:370-83.

\section{Publisher's Note}

Springer Nature remains neutral with regard to jurisdictional claims in published maps and institutional affiliations.

Ready to submit your research? Choose BMC and benefit from:
- fast, convenient online submission
- thorough peer review by experienced researchers in your field
- rapid publication on acceptance
- support for research data, including large and complex data types
- gold Open Access which fosters wider collaboration and increased citations
- maximum visibility for your research: over 100M website views per year
At BMC, research is always in progress.
Learn more biomedcentral.com/submissions

